\title{
THE TRANSFORMATION OF THE ELECTRODYNAMICAL EQUATIONS
}

\author{
By H. Bateman.
}

[Received March 7th, 1909.-Read March 11th, 1909.-Received, in revised form, July 22nd, 1909.]

1. Introduction.

\section{CONTENTS.}

2. The Integral Equations of the Theory of Electrons.

3. The Group of Point Transformations for which the Integral Equations of the Theory of Electrons are Invariant.

4. Spherical Wave Transformations and the Group of Conformal Transformations of a Space of Four Dimensions.

5. The Transformation of Integral Forms.

6. The Invariants of a Spherical Wave Transformation.

7. The Electrodynamical Equations for Ponderable Bodies.

\section{Introduction.}

The numerous experimental and theoretical investigations that have followed Bradley's discovery of aberration have led finally to the enunciation of a principle of relativity. This principle which is being widely used as a working hypothesis is gradually attaining a position analogous to that held by the second law of thermodynamics, ${ }^{*}$ inasmuch as it gives a satisfactory account of the experimental results and is not in direct contradiction with any known facts. +

The mathematical analysis on which the principle is based was introduced by Lorentz in 1892. It consists of a certain space time transformation of the coordinates in which the fundamental equations of the electrodynamical field are unaltered in form.

The principal features of the transformation are the introduction of a local time, and the hypothesis that a body moving through the æther with a constant velocity $v$ suffers a contraction $\sqrt{ }\left(1-v^{2} / c^{2}\right): 1$ in the direction of motion: $c$ being the velocity of light. With the help of this bypothesis

* Cf. H. A. Bumstead, Amer. Jour. of Science, Vol. xxvi (1908).

+ The principle has received additional support from some recent experiments made by Dr. Bucherer, Phys. Zeitschr., Sept., 1908, p. 755. 
a satisfactory explanation of the results of the Michelson-Morley experiment can be given on the supposition of a stationary rther. The results of the experiment seem at first sight to indicate that the æther is carried along with the earth, but on such a supposition a satisfactory explanation of aberration would still be wanting.

The theory of Lorentz's transformation has been developed very con. siderably by Einstein, Planck, and Minkowski. The transformation has become a porwerful instrument of research, inasmuch as it provides a means of transition from the mathematical expressions of physical quantities connected with a system at rest to the corresponding quantities for a similar system in uniform motion. The transformation of Lorentz only enables us to pass from a system at rest to one in uniform motion, but it has been postulated that there is a more general transformation which can be applied to systems moving in a more general manner. The case of a system moving with a constant acceleration has, in fact, been discussed by Einstein.*

The object of the present paper is to find all the transformations for which the electrodynamical equations are invariant. In the case of the simpler equations of the theory of electrons, it is proved that the transformations belong to a certain group which is isomorphic with the group of conformal transformations of a space of four dimensions. It is assumed, however, that the transformation is such that the total charge on a system of particles is unaltered.

I have great pleasure in thanking Mr. E. Cunningham for the stimulus which be gave to this research by the discovery of the formulæ of transformation in the case of an inversion in the four-dimensional space. These formulæ suggested the more general formulæ in terms of Jacobians, and the transition to the integral forms led at once to the present analysis. The two integral forms of the second order had been introduced previously by $\mathrm{Mr}$. Hargreaves in another connection; they enable us to give a very concise expression of the electrodynamical equations which promises to be of considerable importance in future developments. The integrals that occur in the equations differ from the usual surface and volume integrals by the fact that the quantities in the integrand are calculated at different points of space at different times, these times being specified by an arbitrary known law.

The invariance of the two integral forms of the second order leads at once to the formulæ of transformation of the electric and magnetic force, 
and to a number of identical relations which are shown to imply that the differential equation

$$
d x^{2}+d y^{2}+d z^{2}-d t^{2}=0
$$

is invariant. This is the differential equation of the characteristics of the electromagnetic equations and gives the form of an elementary wave front. Since this is a sphere, we have called the group of transformation the group of spherical wave transformations. The differential equation also expresses the condition that two neighbouring particles should be in a position to act on one another. The transformations which leave the electrodynamical equations for ponderable bodies unaltered in form must depend upon the form of the constitutive relations connecting the magnetic induction and electric force with the magnetic force and electric displacement when the bodies are in motion. Minkowski, Einstein, and Laub have proceeded in the opposite way and constructed a set of constitutive relations for bodies in uniform motion, by assuming that the transformations are Lorentzian transformations and transforming the known constitutive relations for bodies at rest.

This method, however, does not give the constitutive relations for the case of a dielectric whose motion is not uniform. The general spherical wave transformation can be applied to obtain a certain type of accelerated motion, but the dimensions and shapes of bodies are continually altering in the transformed system.

An attempt has been made to discover whether there are any types of constitutive relations which are invariant for the general space time transformation. It is shown in $\$ 7$ that it is possible to construct such relations on the assumption that a certain quadratic form is invariant for the transformation. These relations may be supposed to correspond to a special type of configuration and state of motion which preserves its character after any space time transformation, which satisfies certain limitations. The general theory of space time transformations is discussed with the help of two theorems on the transformation of integral forms. It is shown that when a transformation of variables is performed, and the forms equivalent to two given integral forms are known, then the integral forms may be multiplied together by the rules of Grassmann's calculus of extension, and the resulting integral form is equal to the one obtained by multiplying the equivalent integral forms in the same way. Secondly, it is shown that when a quadratic form and its equivalent are known, a pair of equivalent integral forms may be obtained from a given pair by a process of analysis analogous to reciprocation, the new integral forms being of order $n-m$, where $n$ denotes the number of variables and $m$ the order of the original integral forms. These theorems are very

SER. 2. VOL. 8. NO. 1050. 
useful for finding invariants.* Various invariants are calculated with a view of obtaining the general form of the equations of motion. Abrabam's expression of the reaction of radiation on a moving electron is shown to be an invariant for the group of spherical wave transformations, provided it is multiplied by a certain factor.

2. The Integral Equations of the Theory of Electrons.

Let the electromagnetic units be those adopted by Lorentz, and let the units of time and length be so chosen that the velocity of radiation is unity.

Let curl $H$ denote the vector whose components are

$$
\frac{\partial H_{z}}{\partial y}-\frac{\partial H_{y}}{\partial z}, \ldots
$$

when the axes of $x, y, z$ are right-handed and the vector whose components are

$$
\frac{\partial H_{y}}{\partial z}-\frac{\partial H_{z}}{\partial y}, \ldots
$$

when the axes are left-handed. Then the fundamental equationst of the theory of electrons may be written

$$
\left.\begin{array}{rl}
\operatorname{curl} H & =\frac{\partial E}{\partial t}+\rho w \\
\operatorname{curl} E & =-\frac{\partial H}{\partial t} \\
\operatorname{div} E & =\rho \\
\operatorname{div} H & =0
\end{array}\right\},
$$

where $E$ is the electric force, $H$ the magnetic force, $\rho$ the volume density of electricity, and $\rho w$ the convection current.

* They appear to be closely counected with two theorems used by Ricci and Levi Civita, "Méthodes de calcul différential absolu et leurs applications," Math. Ann., Bd. LIv (1901).

$\dagger$ H. A. Lorentz, Ency. d. math. Wiss., Vol. 13, No. 5 ; and Vol. 14, No. 2. The Theory of Electrons, Leipzig (1909), p. 12. 
These equations may be replaced by the two integral equations*

$$
\begin{aligned}
& \iint\left(H_{x} d y d z+H_{y} d z d x+H_{z} d x d y+E_{x} d x d t+E_{y} d y d t+E_{z} d z d t\right)=0 \text {, (II) } \\
& \iint\left(E_{x} d y d z+E_{y} d z d x+E_{y} d x d y-H_{x} d x d t-H_{y} d y d t-H_{z} d z d t\right) \\
& \quad=-\iiint\left(\rho w_{x} d y d z d t+\rho w_{y} d z d x d t+\rho w_{z} d x d y d t-\rho d x d y d z\right) \text {, (III) }
\end{aligned}
$$

provided the integrals receive suitable interpretations. The interpretation that first suggests itself is obtained by regarding $(x, y, z, t)$ as the coordinates of a point in a space of four dimensions. Let any closed twodimensional manifold $S_{2}$ in this space be assigned by equating $x, y, z, t$ to one-valued differentiable functions of two parameters $\alpha, \beta$, and let $\widehat{S}_{2}$ be the boundary of a three-dimensional manifold $S_{3}$ in which the coordinates are like functions of three parameters $\alpha, \beta, \gamma$, of which $\gamma=0$ on $S_{2}$, and $\gamma<0$ on $S_{3}$. Then any term such as $\iint H_{x} d y d z$ may be interpreted to mean $\iint H_{x} \frac{\partial(y, z)}{\partial(\alpha, \beta)} d \alpha d \beta$ taken over $S_{2}$, and any term such as $\iiint \rho d x d y d z$ may be interpreted to mean $\iiint \rho \frac{\partial(x, y, z)}{\partial(\alpha, \beta, \gamma)} d \alpha d \beta d \gamma$ taken over $S_{3}$.

The relations (II) and (III) may now be obtained with the aid of (I) by applying the generalized Green-Stokes theorem as given by Baker, $\dagger$ Poincaré, $\ddagger$ and others.

In order that equations (II) and (III) may be equivalent to (I), the axes must form a right-handed system. If we wish to use left-handed axes we must change the sign of $H$ in (II) and (III).

We shall now endeavour to give a simpler interpretation to the integrals occurring in equations (II) and (III).

Let $S$ be an arbitrary closed surface in the $(x, y, z)$ space, and let $t$ be expressed in terms of $x, y, z$ by an arbitrary law $t=t(x, y, z)$, which must be chosen, however, in such a way that $t$ is a single-valued function which is finite together with its derivatives with regard to $x, y, z$ at all points within $S$ and on $S$ itself. Let the coordinates of points on $S$ be expressed in terms of two parameters $\alpha, \beta$.

* The integral forms occurring in these equations have been studied by Hargreaves, Camb. Phil. Trans., Vol. 21, p. 107 (1908).

$\dagger$ Camb. Phil. Trans., Vol. 18 (1900), p. 408.

$\ddagger$ Acta Math., t. 9 (1887), p. 321. 
A term such as $\iint E_{x} d x d t$ may now be understood to mean*

$$
\iint E_{x}\left[\frac{\partial(x, t)}{\partial(y, z)} \frac{\partial(y, z)}{\partial(\alpha, \beta)}+\frac{\partial(x, t)}{\partial(z, x)} \frac{\partial(z, x)}{\partial(\alpha, \beta)}+\frac{\partial(x, t)}{\partial(x, y)} \frac{\partial(x, y)}{\partial(\alpha, \beta)}\right] d \alpha d \beta,
$$

and a term such as $\iiint \rho w_{x} d y d z d t$ to mean

$$
\iiint \rho w_{x^{\prime}} \frac{\partial(y, z, t)}{\partial(x, y, z)} d x d y d z
$$

The equation (II) may now be written

$$
\begin{aligned}
& \iint\left[\left(E_{x}-H_{u} \frac{\partial t}{\partial z}+H_{z} \frac{\partial t}{\partial y}\right) d y d z\right.+\left(E_{y}-H_{z} \frac{\partial t}{\partial x}+H_{x} \frac{\partial t}{\partial z}\right) d z d x \\
&\left.+\left(E_{z}-H_{x} \frac{\partial t}{\partial y}+H_{y} \frac{\partial t}{\partial x}\right) d x d y\right] \\
&=-\iiint\left(\rho w_{x} \frac{\partial t}{\partial x}+\rho w_{y} \frac{\partial t}{\partial y}+\rho v \div \frac{\partial t}{\partial z}-\rho\right) d x d y d z .
\end{aligned}
$$

Transforming the left-hand side by means of Green's theorem, we get

$$
\begin{gathered}
\iiint\left[\frac{d}{d x}\left(E_{x}-H_{y} \frac{\partial t}{\partial z}+H_{z} \frac{\partial t}{\partial y}\right)+\frac{d}{d y}\left(E_{y}-H_{:} \frac{\partial t}{\partial x}+H_{x} \frac{\partial t}{\partial z}\right)\right. \\
\left.+\frac{d}{d z}\left(E_{z}-H_{x} \frac{\partial t}{\partial y}+H_{y} \frac{\partial t}{\partial x}\right)\right] d x d y d z \\
\frac{d E_{x}}{d x}=\frac{\partial E_{x}}{\partial x}+\frac{\partial E_{x}}{\partial t} \frac{\partial t}{\partial x} \\
\frac{d H_{y}}{d x}=\frac{\partial H_{y}}{\partial x}+\frac{\partial H_{y}}{\partial t} \frac{\partial t}{\partial x}
\end{gathered}
$$

Now

hence the above integral may be written

$$
\begin{aligned}
& \iiint\left[\left(\frac{\partial E_{z}}{\partial t}-\frac{\partial H_{z}}{\partial y}+\frac{\partial H_{y}}{\partial z}\right) \frac{\partial t}{\partial x}+\left(\frac{\partial E_{y}}{\partial t}-\frac{\partial H_{x}}{\partial z}+\frac{\partial H_{z}}{\partial x}\right) \frac{\partial t}{\partial y}\right. \\
& \left.+\left(\frac{\partial E_{z}}{\hat{\imath} t}-\frac{\partial H_{y}}{\partial x}+\frac{\hat{\partial} H_{x}}{\partial y}\right) \frac{\partial t}{\partial z}+\left(\frac{\partial E_{x}}{\partial x}+\frac{\partial E_{y}}{\partial y}+\frac{\partial E_{z}}{\partial z}\right)\right] d x d y d z, \\
& \text { * The relation } \frac{\partial(x, t)}{\partial(u, \beta)}=\frac{\partial(x, t)}{\partial(y, z)} \frac{\partial(y, z)}{\partial(\alpha, \beta)}+\frac{\partial(x, t) \partial(z, x)}{\partial(z, x) \partial(\alpha, \beta)}+\frac{\partial(x, t)}{\partial(x, y)} \frac{\partial(x, y)}{\partial(\alpha, \beta)}
\end{aligned}
$$

is a particular case of a general relation given in Scott's Determinants, p. 134. 
and this is equivalent to

$$
-\iiint\left(\rho w_{x} \frac{\partial t}{\partial x}+\rho w_{y} \frac{\partial t}{\partial y}+\rho w_{z} \frac{\partial t}{\partial z}-\rho\right) d x d y d z
$$

if equations (I) are satisfied and the axes form a right-handed system. In the same way, it can be shown that the integral on the left-hand side of (II) is zero, if equations (I) are satisfied.

Conversely, if equations (II) and (III) are satisfied for every closed surface $S$ and for every law $t=t(x, y, z)$, such that $\partial t / \partial x, \partial t / \partial y, \partial t / c z$ are finite and continuous within $S$ and on its surface, then equations (I) are also satisfied, provided, of course, that the quantities $E_{x}, \ldots, H_{x}, \ldots$ possess derivatives which behave in such a way that an application of Green's theorem is permissible.

It seems natural to regard equations (II) and (III) as the fundamental equations of the theory of electrons, because they do not require the assumptions that the medium is continuous and that vectors $E, H$ can be associated with every point in space ;* all that is required is that the quantities $E_{x}, \ldots, \rho w_{x}, \ldots$ shall be integrable.

It is clear that in the general case the quantities occurring in these equations are evaluated at different points of space at different times. The integrals are thus more general than the usual surface and volume integrals, and seem to be better adapted for purposes of measurement, the difficulty of measuring quantities at different points of space at the same time being avoided.

Equation (III) may be regarded as the definition of the electric charge associated with a system of particles. The triple integral represents the total charge on the particles. $\dagger$ Each particle is supposed to be within

* In the case of a single pulse travelling across a medium in which the vectors $E$ and $H$ are initially zero, these vectors may exist at a given point at one time and not at another.

$\dagger$ This may be proved by showing that the triple integral remains invariant during the motion of a system of electrons.

Let an electron which was at the point $(x, y, z)$ at time $t$ be in a new position $\left(x^{\prime}, y^{\prime}, z^{\prime}\right)$ at time $t^{\prime}$, where

$$
\begin{array}{ll}
x^{\prime}=x+\epsilon w_{x}, & z^{\prime}=z+\epsilon w_{x}, \\
y^{\prime}=y+\epsilon w_{y}, & t^{\prime}=t+\epsilon,
\end{array}
$$

and $\epsilon$ is a small quantity which is a funotion of $x, y, z, t$. The integral form is transformed into

$$
\rho^{\prime} w_{x}^{\prime} d y^{\prime} d z^{\prime} d t^{\prime}+\rho^{\prime} w_{y}^{\prime} d z^{\prime} d x^{\prime} d t^{\prime}+\rho^{\prime} v_{z}^{\prime} d x^{\prime} d y^{\prime} d t^{\prime}-\rho^{\prime} d x^{\prime} d y^{\prime} d z^{\prime},
$$

where

$$
\rho w_{x}=\rho^{\prime} w_{x}^{\prime} \frac{\partial\left(y^{\prime}, z^{\prime}, t\right)}{\partial(y, z, t)}+\rho^{\prime} w_{y}^{\prime} \frac{\partial\left(z^{\prime}, x^{\prime}, t^{\prime}\right)}{\partial(y, z, t)}+\rho^{\prime} w_{=}^{\prime} \frac{\partial\left(x^{\prime}, y^{\prime}, t\right)}{\partial(y, z, t)}-\rho^{\prime} \frac{\partial\left(x^{\prime}, y^{\prime}, z^{\prime}\right)}{\partial(y, z, t)},
$$


the closed surface $S$ at the moment when its charge is evaluated, but since the charges on the particles are evaluated at different times, the particles need not all be within the closed surface at a given time. This explains why the total charge is not

$$
\iiint \rho d x d y d z
$$

The double integral expresses the total charge as a surface integral, but here again the particles over which the integration extends are not all on the surface at the same time, but at different times.

In the same way equation (II) may be regarded as a general way of expressing the fact that there is no free magnetism.

If $\Phi$ is the scalar electromagnetic potential and $\left(A_{x}, A_{y}, A_{z}\right)$ the components of the electromagnetic vector potential, we have

$$
E_{x}=-\frac{\partial \Phi}{\partial x}-\frac{\partial A_{x}}{\partial t}, \quad H_{x}=\frac{\partial A_{x}}{\partial y}-\frac{\partial A_{y}}{\partial z} .
$$

or

$$
\rho^{\prime} w_{x}^{\prime} \frac{\partial\left(x^{\prime}, y^{\prime}, z^{\prime}, t^{\prime}\right)}{\partial(x, y, z, t)}=\rho w_{x} \frac{\partial x^{\prime}}{\partial x}+\rho w_{y} \frac{\partial x^{\prime}}{\partial y}+\rho w_{z} \frac{\partial x^{\prime}}{\partial z}+\rho \frac{\partial x^{\prime}}{\partial t}
$$

This gives

$$
\begin{aligned}
& \rho^{\prime} w_{x}^{\prime} \frac{\partial\left(x^{\prime}, y^{\prime}, z^{\prime}, t^{\prime}\right)}{\partial(x, y, z, t)}=\rho w_{x}\left[1+\epsilon-\frac{\partial w_{x}}{\partial x}+v_{x} \frac{\partial \epsilon}{\partial x}\right]+\rho v_{y}\left[\epsilon \frac{\partial w_{x}}{\partial y}+w_{x} \frac{\partial^{\epsilon}}{\partial y}\right] \\
& +\rho w_{z}\left[\epsilon \frac{\partial w_{x}}{\partial z}+w_{x} \frac{\partial \epsilon}{\partial z}\right]+\rho\left[\epsilon \frac{\partial w_{x}}{\partial t}+w_{x} \frac{\partial \epsilon}{\partial t}\right] \text {, } \\
& \rho^{\prime} \frac{\partial\left(x^{\prime}, y^{\prime}, z^{\prime}, t^{\prime}\right)}{\partial(x, y, z, t)}=\rho w_{x} \frac{\partial \epsilon}{\partial x}+\rho 2 v_{y} \frac{\partial \epsilon}{\partial y}+\rho w_{z} \frac{\partial \epsilon}{\partial z}+\rho\left[1+\frac{\partial \epsilon}{\partial t}\right] .
\end{aligned}
$$

Multiplying the last equation by $w_{s}$, and subtracting from the first, we get

$$
\rho^{\prime} \frac{\partial\left(x^{\prime}, y^{\prime}, z^{\prime}, t^{\prime}\right)}{\partial(x, y, z, t)}\left(w_{x}^{\prime}-w_{x}\right)=\rho \epsilon\left[\frac{\partial w_{x}}{\partial t}+w_{x} \frac{\partial w_{x}}{\partial x}+v_{y} \frac{\partial w_{s}}{\partial y}+w v_{z} \frac{\partial w_{x}}{\partial z}\right]
$$

Putting

$$
\frac{d}{d t}=\frac{\partial}{\partial t}+w_{x} \frac{\partial}{\partial x}+w_{y} \frac{\partial}{\partial y}+w_{z} \frac{\partial}{\partial z}
$$

we see that the equation is satisfied if

$$
\left(1+\frac{d \epsilon}{d t}\right)\left(w_{x}^{\prime}-w_{x}\right)=\epsilon \frac{d w_{x}}{d t},
$$

and if $\frac{d \epsilon}{d t}$ is a small quantity of the first order

$$
w_{x}^{\prime}-w_{x}=\epsilon \frac{d w_{s}}{d t}
$$

in other words $\left(w_{x}^{\prime}, w_{y}^{\prime}, w_{z}^{\prime}\right)$ are the component velocities at time $t+\epsilon$.

Since the integral form is an invariant, we may calculate its value by considering the swarm of electrons at a given time $t$. The temporal terms then disappear, and the integral reduces simply to $-\rho d x d y d z$, i.e., the total negative charge on the system of electrons. 
These equations are equivalent to the single integral equation

$$
\begin{aligned}
& \int\left(A_{x} d x+A_{y} d y+A_{z} d z-\Phi d t\right) \\
& \quad=\iint\left(H_{y} d y d z+H_{y} d z d x+H_{z} d x d y+E_{x} d x d t+E_{y} d y d t+E_{z} d z d t\right),(\mathrm{IV})
\end{aligned}
$$

provided a suitable interpretation is given to the integrals.

Take any closed curve $C$ and a surface $\Sigma$ bounded by this curve. Let $t$ be expressed in terms of $(x, y, z)$ by an arbitrary known law; then the line integral may be understood to mean

$$
\int\left[\left(A_{x}-\Phi \frac{\partial t}{\partial x}\right) d x+\left(A_{y}-\Phi \frac{\partial t}{\partial y}\right) d y+\left(A_{j}-\Phi \frac{\partial t}{\partial z}\right) d z\right],
$$

and can be transformed into the surface integral by means of Stokes's theorem. Since

$$
\begin{aligned}
\frac{d}{d y}\left(A_{z}-\Phi \frac{\partial t}{\partial z}\right)-\frac{d}{d z} & \left(A_{y}-\Phi \frac{\partial t}{\partial y}\right) \\
& =\frac{\partial A_{z}}{\partial y}-\frac{\partial A_{y}}{\partial z}+\left(\frac{\partial A_{z}}{\partial t}+\frac{\partial \Phi}{\partial z}\right) \frac{\partial t}{\partial y}-\left(\frac{\partial A_{y}}{\partial t}+\frac{\partial \Phi}{\partial y}\right) \frac{\partial t}{\partial z} \\
& =H_{x}-E_{z} \frac{\partial t}{\partial y}+E_{l} \frac{\partial t}{\partial z},
\end{aligned}
$$

the surface integral which is obtained is

$$
\begin{array}{r}
\iint\left[\left(H_{x}-E_{z} \frac{\partial t}{\partial y}+E_{y} \frac{\partial t}{\partial z}\right) d y d z\right. \\
+\left(H_{y}-E_{x} \frac{\partial t}{\partial z}+E_{z} \frac{\partial t}{\partial x}\right) d z d x \\
\left.+\left(H_{z}-E_{y} \frac{\partial t}{\partial x}+E_{x} \frac{\partial t}{\partial y}\right) d x d y\right], \\
\text { or } \quad \iint\left(H_{x} d y d z+H_{y} d z d x+H_{z} d x d y+E_{x} d x d t+E_{y} d y d t+E_{z} d z d t\right) .
\end{array}
$$

Hence equation (IV) is established.

3. The Group of Point Transformations for which the Integral Equations of the Theory of Electrons are Invariant.

Let us consider a transformation of coordinates from $(x, y, z, t)$ to $\left(x^{\prime}, y^{\prime}, z^{\prime}, t^{\prime}\right)$ which is biuniform within a certain domain of values of $(x, y, z, t)$. We shall suppose that the choice of a transformation is 
limited by the condition that the total charge on a system of particles is invariant. This is expressed analytically by the equation

$\rho w_{x} d y d z d t+\rho w_{y} d z d x d t+\rho w_{z} d x d y d t-\rho d x d y d z$

$$
=\rho^{\prime} w_{x}^{\prime} d y^{\prime} d z^{\prime} d t^{\prime}+\rho^{\prime} w_{y}^{\prime} d z^{\prime} d x^{\prime} d t^{\prime}+\rho^{\prime} w_{z}^{\prime} d x^{\prime} d y^{\prime} d t^{\prime}-\rho^{\prime} d x^{\prime} d y^{\prime} d z^{\prime},
$$

provided the axes form a right-handed system in each case.

If the transformation is such that the integral equations of the theory of electrons are invariant, we must have

$E_{x} d y d z+E_{y} d z d x+E_{z} d x d y-H_{x} d x d t-H_{y} d y d t-H_{z} d z d t$

$=E_{x}^{\prime} d y^{\prime} d z^{\prime}+E_{y}^{\prime} d z^{\prime} d x^{\prime}+E_{z}^{\prime} d x^{\prime} d y^{\prime}-H_{x}^{\prime} d x^{\prime} d t^{\prime}-H_{y}^{\prime} d y^{\prime} d t^{\prime}-H_{z}^{\prime} d z^{\prime} d t^{\prime}$,

$H_{x} d y d z+H_{y} d z d x+H_{z} d x d y+E_{x} d x d t+E_{y} d y d t+E_{z} d z d t$

$=\theta\left[H_{x}^{\prime} d y^{\prime} d z^{\prime}+H_{y}^{\prime} d z^{\prime} d x^{\prime}+H_{z}^{\prime} d x^{\prime} d y^{\prime}+E_{x}^{\prime} d x^{\prime} d t^{\prime}+E_{y}^{\prime} d y^{\prime} d t^{\prime}+E_{z}^{\prime} d z^{\prime} d t^{\prime}\right]$.

where $\theta$ is a constant.

These relations give two sets of equations connecting the quantities $E_{x}, \ldots, H_{x}, \ldots$ with $E_{x}^{\prime}, \ldots, H_{x}^{\prime}, \ldots$, viz.,

$$
\begin{array}{r}
E_{x}=E_{x}^{\prime} \frac{\partial\left(y^{\prime}, z^{\prime}\right)}{\partial(y, z)}+E_{y}^{\prime} \frac{\partial\left(z^{\prime}, x^{\prime}\right)}{\partial(y, z)}+E_{z}^{\prime} \frac{\partial\left(x^{\prime}, y^{\prime}\right)}{\partial(y, z)}-H_{x}^{\prime} \frac{\partial\left(x^{\prime}, t^{\prime}\right)}{\partial(y, z)} \\
-H_{y}^{\prime} \frac{\partial\left(y^{\prime}, t^{\prime}\right)}{\partial(y, z)}-H_{z}^{\prime} \frac{\partial\left(z^{\prime}, t^{\prime}\right)}{\partial(y, z)}, \\
-H_{x}=E_{x}^{\prime} \frac{\partial\left(y^{\prime}, z^{\prime}\right)}{\partial(x, t)}+E_{y}^{\prime} \frac{\partial\left(z^{\prime}, x^{\prime}\right)}{\partial(x, t)}+E_{z}^{\prime} \frac{\partial\left(x^{\prime}, y^{\prime}\right)}{\partial(x, t)}-H_{x}^{\prime} \frac{\partial\left(x^{\prime}, t^{\prime}\right)}{\partial(x, t)} \\
-H_{y}^{\prime} \frac{\partial\left(y^{\prime}, t^{\prime}\right)}{\partial(x, t)}-H_{z}^{\prime} \frac{\partial\left(z^{\prime}, t^{\prime}\right)}{\partial(x, t)},
\end{array}
$$

and

$$
\begin{aligned}
& H_{x}=\theta\left[\quad H_{x}^{\prime} \frac{\partial\left(y^{\prime}, z^{\prime}\right)}{\partial(y, z)}+H_{y}^{\prime} \frac{\partial\left(z^{\prime}, x^{\prime}\right)}{\partial(y, z)}+H_{z}^{\prime} \frac{\partial\left(x^{\prime}, y^{\prime}\right)}{\partial(y, z)}\right. \\
& \left.+E_{x}^{\prime} \frac{\partial\left(x^{\prime}, t^{\prime}\right)}{\partial(y, z)}+E_{y}^{\prime} \frac{\partial\left(y^{\prime}, t^{\prime}\right)}{\partial(y, z)}+E_{z}^{\prime} \frac{\partial\left(z^{\prime}, t^{\prime}\right)}{\partial(y, z)}\right], \\
& E_{x}=\theta\left[H_{x}^{\prime} \frac{\partial\left(y^{\prime}, z^{\prime}\right)}{\partial(x, t)}+H_{y}^{\prime} \frac{\partial\left(z^{\prime}, x^{\prime}\right)}{\partial(x, t)}+H_{z}^{\prime} \frac{\partial\left(x^{\prime}, y^{\prime}\right)}{\partial(x, t)}\right. \\
& \left.+E_{x}^{\prime} \frac{\partial\left(x^{\prime}, t^{\prime}\right)}{\partial(x, t)}+E_{y}^{\prime} \frac{\partial\left(y^{\prime}, t^{\prime}\right)}{\partial(x, t)}+E_{z}^{\prime} \frac{\partial\left(z^{\prime}, t^{\prime}\right)}{\partial(x, t)}\right] .
\end{aligned}
$$

In order that these equations may be equivalent to one another* we must

* It is assumed here that the equations of transformation are independent of $E_{x}, E_{y}, E_{z}$, $H_{x}, H_{v}, H_{z}$. 
1909.] The transformation of the electrodynamical equations. 283 have $\theta^{2}=1$ and eighteen relations of the types

$$
\begin{aligned}
& \frac{\partial\left(y^{\prime}, z^{\prime}\right)}{\partial(x, y)}=\theta \frac{\partial\left(x^{\prime}, t^{\prime}\right)}{\partial(z, t)}, \\
& \frac{\partial\left(y^{\prime}, z^{\prime}\right)}{\partial(x, t)}=-\theta \frac{\partial\left(x^{\prime}, t^{\prime}\right)}{\partial(y, z)} .
\end{aligned}
$$

There are clearly nine relations of the first type and nine relations of the second type. We shall now show that when $\theta^{2}=1$ these relations imply that there is a relation of the form

$$
d x^{\prime 2}+d y^{\prime 2}+d z^{\prime 2}-d t^{2}=\lambda^{2}\left(d x^{2}+d y^{2}+d z^{2}-d t^{2}\right) ;
$$

for this purpose we shall require the following lemma.

Lemma.-Let the sixteen quantities

$$
\left(\alpha_{1} \alpha_{2} \alpha_{3} \alpha_{4}\right), \quad\left(\beta_{1} \beta_{2} \beta_{3} \beta_{4}\right), \quad\left(\gamma_{1} \gamma_{2} \gamma_{3} \gamma_{4}\right), \quad\left(\delta_{1} \delta_{2} \delta_{3} \delta_{4}\right)
$$

be connected by the eighteen relations of type

$$
\begin{gathered}
\beta_{2} \gamma_{8}-\beta_{8} \gamma_{2}=a_{1} \delta_{4}-a_{4} \delta_{1}, \quad \gamma_{2} \alpha_{8}-\gamma_{3} \alpha_{2}=\beta_{1} \delta_{4}-\beta_{4} \delta_{1}, \\
a_{2} \beta_{3}-a_{3} \beta_{2}=\gamma_{1} \delta_{4}-\gamma_{4} \delta_{1},
\end{gathered}
$$

which imply that conjugate minors of the determinant $\left[a_{1} \beta_{2} \gamma_{3} \delta_{4}\right]$ are equal. The identity

$$
\alpha_{1}\left(\alpha_{2} \beta_{3}-\alpha_{3} \beta_{2}\right)+\alpha_{2}\left(\alpha_{3} \beta_{1}-a_{1} \beta_{3}\right)+\alpha_{3}\left(\alpha_{1} \beta_{2}-a_{2} \beta_{1}\right)=0
$$

then gives $a_{1}\left(\gamma_{1} \delta_{4}-\gamma_{4} \delta_{1}\right)+a_{2}\left(\gamma_{2} \delta_{4}-\gamma_{4} \delta_{2}\right)+a_{3}\left(\gamma_{3} \delta_{4}-\gamma_{4} \delta_{3}\right)=0$ or

$$
\delta_{4}\left(a_{1} \gamma_{1}+a_{2} \gamma_{2}+a_{8} \gamma_{3}+a_{4} \gamma_{4}\right)=\gamma_{4}\left(a_{1} \delta_{1}+a_{2} \delta_{2}+a_{3} \delta_{3}+a_{4} \delta_{4}\right) .
$$

Introducing the notation

$$
(\alpha \gamma) \equiv \alpha_{1} \gamma_{1}+\alpha_{2} \gamma_{2}+\alpha_{3} \gamma_{3}+\alpha_{4} \gamma_{4},
$$

we may obtain in the above way the equations

$$
\begin{array}{ll}
\delta_{1}(\alpha \gamma)=\gamma_{1}(\alpha \delta), & \delta_{3}(\alpha \gamma)=\gamma_{3}(\alpha \delta), \\
\delta_{2}(\alpha \gamma)=\gamma_{2}(\alpha \delta), & \delta_{4}(\alpha \gamma)=\gamma_{4}(\alpha \delta) ; \\
\delta_{1}(\beta \gamma)=\gamma_{1}(\beta \delta), & \delta_{3}(\beta \gamma)=\gamma_{3}(\beta \delta), \\
\delta_{2}(\beta \gamma)=\gamma_{2}(\beta \delta), & \delta_{4}(\beta \gamma)=\gamma_{4}(\beta \delta) .
\end{array}
$$

Hence, either

$$
(\alpha \gamma)=(\alpha \delta)=(\beta \gamma)=(\beta \delta)=0
$$

or all the quantities of type $\gamma_{1} \delta_{2}-\gamma_{2} \delta_{1}$ are zero. In the same way we can prove that either

$$
(\alpha \beta)=(\alpha \delta)=(\gamma \beta)=(\gamma \delta)=0
$$


or all the quantities of type $\beta_{1} \delta_{2}-\beta_{2} \delta_{1}$ are zero, and that either

$$
(\beta a)=(\beta \delta)=(\gamma \alpha)=(\gamma \delta)=0
$$

or all the quantities of type $\alpha_{1} \delta_{2}-\alpha_{2} \delta_{1}$ are zero. It follows from this that either (1) the six quantities $(\alpha \beta)$ are zero or (2) that the thirty-six quantities $\left(\alpha_{1} \delta_{2}-a_{2} \delta_{1}\right),\left(\beta_{1} \delta_{2}-\beta_{2} \delta_{1}\right),\left(\gamma_{1} \delta_{2}-\gamma_{2} \delta_{1}\right), \ldots$ are all zero or (3) that there is a set of relations

$$
\begin{gathered}
(a \gamma)=(\alpha \delta)=(\beta \gamma)=(\beta \delta)=0, \\
\frac{\beta_{1}}{\delta_{1}}=\frac{\beta_{2}}{\delta_{3}}=\frac{\beta_{3}}{\delta_{3}}=\frac{\beta_{4}}{\delta_{4}}, \quad \frac{\alpha_{1}}{\delta_{1}}=\frac{a_{2}}{\delta_{2}}=\frac{a_{3}}{\delta_{3}}=\frac{u_{4}}{\delta_{4}} .
\end{gathered}
$$

It is easy to see, however, that in the latter case we also have

$$
(\gamma \delta)=(\alpha \beta)=0 \text {. }
$$

Hence, in all cases,

$$
(\alpha \gamma)=(\alpha \beta)=(\alpha \delta)=(\beta \gamma)=(\beta \delta)=(\gamma \delta)=0 .
$$

Again, we have

$$
\left|\begin{array}{ccc}
a_{1} & \beta_{1} & \gamma_{1} \\
a_{2} & \beta_{2} & \gamma_{2} \\
a_{3} & \dot{\beta_{3}} & \gamma_{3}
\end{array}\right|=\alpha_{1}\left(a_{1} \delta_{4}-a_{4} \delta_{1}\right)+\alpha_{2}\left(a_{3} \delta_{4}-a_{4} \delta_{2}\right)+a_{3}\left(a_{3} \delta_{4}-a_{4} \delta_{3}+a_{4}^{2}\right)-a_{4}\left(a_{1} \delta_{1}+a_{2} \delta_{2}+a_{3} \delta_{3}+a_{4} \delta_{4}\right) ;
$$

hence, since the last term is zero,

This gives

$$
\left|\begin{array}{lll}
\alpha_{1} & \beta_{1} & \gamma_{1} \\
\alpha_{2} & \beta_{2} & \gamma_{2} \\
\alpha_{3} & \beta_{3} & \gamma_{3}
\end{array}\right|=\delta_{4}\left(a_{1}^{2}+a_{2}^{2}+\alpha_{3}^{2}+\alpha_{4}^{2}\right)
$$

$$
\left(a_{1}^{2}+u_{2}^{2}+a_{3}^{2}+a_{4}^{2}\right)\left(\delta_{1}^{2}+\delta_{2}^{2}+\delta_{3}^{2}+\delta_{4}^{2}\right)=\left|\begin{array}{cccc}
a_{1} & \beta_{1} & \gamma_{1} & \delta_{1} \\
u_{2} & \beta_{2} & \gamma_{2} & \delta_{2} \\
u_{3} & \beta_{3} & \gamma_{3} & \delta_{3} \\
a_{4} & \beta_{4} & \gamma_{4} & \delta_{4}
\end{array}\right|=\lambda^{4},
$$

say, and there are similar equations in $\alpha, \beta, \alpha \gamma, \beta \gamma, \beta \delta, \gamma \delta$. It follows that

$$
\begin{aligned}
a_{1}^{2}+a_{2}^{2}+a_{3}^{2}+\alpha_{4}^{2} & =\beta_{1}^{2}+\beta_{2}^{2}+\beta_{3}^{2}+\beta_{4}^{2} \\
& =\gamma_{1}^{2}+\gamma_{2}^{2}+\gamma_{3}^{2}+\gamma_{4}^{2}=\delta_{1}^{2}+\delta_{2}^{2}+\delta_{3}^{2}+\delta_{4}^{2}= \pm \lambda^{2} .
\end{aligned}
$$

These conditions, combined with the previous set, imply that the sixteen quantities $\alpha_{r}, \beta_{s}, \ldots$ are the elements of an orthogonal matrix. 
If the signs of the $\alpha$ 's are all changed, the relations take the form

$$
\beta_{2} \gamma_{3}-\beta_{3} \gamma_{2}=-\left(a_{1} \delta_{4}-a_{4} \delta_{1}\right) \text {, }
$$

but the final result is unaltered; hence a set of relations of this type also imply that the quantities $\alpha$ are the elements of an orthogonal matrix.

Taking the quantities $\alpha, \beta, \ldots$ to be the elements of the matrix

$$
\left|\begin{array}{llll}
\frac{\partial x^{\prime}}{\partial x} & \frac{\partial x^{\prime}}{\partial y}, & \frac{\partial x^{\prime}}{\partial z} & \frac{\partial x^{\prime}}{i \partial t} \\
\frac{\partial y^{\prime}}{\partial x} & \frac{\partial y^{\prime}}{\partial y} & \frac{\partial y^{\prime}}{\partial z} & \frac{\partial y^{\prime}}{i \partial t} \\
\frac{\partial z^{\prime}}{\partial x} & \frac{\partial z^{\prime}}{\partial y} & \frac{\partial z^{\prime}}{\partial z} & \frac{\partial z^{\prime}}{i \partial t} \\
i \frac{\partial t^{\prime}}{\partial x} & i \frac{\partial t^{\prime}}{\partial y} & i \frac{\partial t^{\prime}}{\partial z} & \frac{\partial t^{\prime}}{\partial t}
\end{array}\right|
$$

we have the result that, if eighteen relations of the type

$$
\frac{\partial\left(y^{\prime}, z^{\prime}\right)}{\partial(x, y)}=\frac{\partial\left(x^{\prime}, t^{\prime}\right)}{\partial(z, t)}, \quad \frac{\partial\left(y^{\prime}, z^{\prime}\right)}{\partial(x, t)}=-\frac{\partial\left(x^{\prime}, t^{\prime}\right)}{\partial(y, z)}
$$

or of the type $\frac{\partial\left(y^{\prime}, z^{\prime}\right)}{\partial(x, y)}=-\frac{\partial\left(x^{\prime}, t^{\prime}\right)}{\partial(z, t)}, \quad \frac{\partial\left(y^{\prime}, z^{\prime}\right)}{\partial(x, t)}=\frac{\partial\left(x^{\prime}, t^{\prime}\right)}{\partial(y, z)}$

are satisfied, then the matrix is an orthogonal one, and this implies that

$$
d x^{\prime 2}+d y^{\prime 2}+d z^{\prime 2}-d t^{\prime 2}=\lambda^{2}\left[d x^{2}+d y^{2}+d z^{2}-d t^{2}\right] ;
$$

in other words, that the differential equation

$$
(d x)^{2}+(d y)^{2}+(d z)^{2}-(d t)^{2}=0
$$

is an invariant. The converse is also true, and may be easily verified.

This differential equation expresses the condition that two neighbouring particles should be in a position to act on one another; it also indicates that an elementary wave starting from a given point will have the form of a sphere. For this reason we shall call a transformation which leaves the differential equation invariant, a spherical wave transformation. Our analysis shows that a transformation which leaves the integral equations of the theory of electrons invariant is necessarily a spherical wave transformation. We must next inquire whether all spherical wave transformations are relevant for our purpose. If we call $t^{\prime}$ the local time, we must exclude transformations which make the local time run backwards as $t$ increases. Hence, in order that a transformation may be relevant, the condition

$$
\frac{\partial t^{\prime}}{\partial t}>0
$$

must be satisfied. 
Secondly, we must exclude transformations in which the axes are changed from a right-handed system to a left-handed system, for then equation (1) would imply a change from positive electricity to negative electricity. Snch a transformation, however, becomes relevant when equation (1) is replaced by

$\rho w_{x} d y d z d t+\rho w_{y} d z d x d t+\rho v v_{z} d x d y d t-\rho d x d y d z$

$$
=-\rho^{\prime} w_{x}^{\prime} d y^{\prime} d z^{\prime} d t^{\prime}-\rho^{\prime} w_{y}^{\prime} d z^{\prime} d x^{\prime} d t^{\prime}-\rho^{\prime} w_{z}^{\prime} d x^{\prime} d y^{\prime} d t^{\prime}+\rho^{\prime} d x^{\prime} d y^{\prime} d z^{\prime},
$$

equation (2) by

$E_{x} d y d z+E_{y} d z d x+E_{s} d x d y-H_{x} d x d t-H_{y} d y d t-H_{z} d z d t$

$=-E_{x}^{\prime} d y^{\prime} d z^{\prime}-E_{y}^{\prime} d z^{\prime} d x^{\prime}-E_{z}^{\prime} d x^{\prime} d y^{\prime}-H_{x}^{\prime} d x^{\prime} d t^{\prime}-H_{y}^{\prime} d y^{\prime} d t^{\prime}-H_{z}^{\prime} d z^{\prime} d t^{\prime}$,

and equation (3) by

$$
\begin{aligned}
& H_{x} d y d z+H_{y} d z d x+H_{z} d x d y+E_{x} d x d t+E_{y} d y d t+E_{z} d z d t \\
& \quad=\theta\left[-H_{x}^{\prime} d y^{\prime} d z^{\prime}-H_{y}^{\prime} d z^{\prime} d x^{\prime}-H_{z}^{\prime} d x^{\prime} d y^{\prime}+E_{x}^{\prime} d x^{\prime} d t^{\prime}+E_{y}^{\prime} d y^{\prime} d t^{\prime}+E_{z}^{\prime} d z^{\prime} d t^{\prime}\right]
\end{aligned}
$$

These relations also imply that the transformation is a spherical wave transformation, and so a transformation which changes a right-handed system of axes into a left-handed system is relevant, but the formulæ of transformation of the components of the electric and magnetic force are not the same as before. The sign of $\theta$ will be determined later by the condition that a right-handed set of axes is transformed into a lefthanded set. If

$$
\frac{\partial\left(x^{\prime}, y^{\prime}, z^{\prime}\right)}{\partial(x, y, z)}>0
$$

corresponding sets of axes are both right-handed or both left-handed. If, on the other hand,

$$
\frac{\partial\left(x^{\prime}, y^{\prime}, z^{\prime}\right)}{\partial(x, y, z)}<0
$$

the axes are right-handed in one system and left-handed in the other.

It is easy to establish a relation between the two quantities

$$
\begin{gathered}
\frac{\partial t^{\prime}}{\partial t} \text { and } \frac{\partial\left(x^{\prime}, y^{\prime}, z^{\prime}\right)}{\partial(x, y, z)} \\
\frac{\partial\left(y^{\prime}, z^{\prime}\right)}{\partial(y, z)}=\theta \frac{\partial\left(x^{\prime}, t^{\prime}\right)}{\partial(x, t)}, \quad \frac{\partial\left(z^{\prime}, x^{\prime}\right)}{\partial(y, z)}=\theta \frac{\partial\left(y^{\prime}, t^{\prime}\right)}{\partial(x, t)} \\
\frac{\partial\left(x^{\prime}, y^{\prime}\right)}{\partial(y, z)}=\theta \frac{\partial\left(z^{\prime}, t^{\prime}\right)}{\partial(x, t)}
\end{gathered}
$$

Since 


\section{we have}

$$
\begin{gathered}
\begin{aligned}
\frac{\partial\left(x^{\prime}, y^{\prime}, z^{\prime}\right)}{\partial(x, y, z)}=\theta\left[\frac { \partial t ^ { \prime } } { \partial t } \left\{\left(\frac{\partial x^{\prime}}{\partial x}\right)^{2}\right.\right. & \left.+\left(\frac{\partial y^{\prime}}{\partial x}\right)^{2}+\left(\frac{\partial z^{\prime}}{\partial x}\right)^{2}-\left(\frac{\partial t^{\prime}}{\partial x}\right)^{2}\right\} \\
& -\frac{\partial x^{\prime}}{\partial x}\left\{\frac{\partial x^{\prime}}{\partial x} \frac{\partial x^{\prime}}{\partial t}+\frac{\partial y^{\prime}}{\partial x} \frac{\partial y^{\prime}}{\partial t}+\frac{\partial z^{\prime}}{\partial x} \frac{\partial z^{\prime}}{\partial t}-\frac{\partial t^{\prime}}{\partial x} \frac{\left.\partial t^{\prime}\right)}{\partial t} ;\right] \\
\text { or } \quad \frac{\partial\left(x^{\prime}, y^{\prime}, z^{\prime}\right)}{\partial(x, y, z)}=\theta \frac{\partial t^{\prime}}{\partial t} & {\left[\left(\frac{\partial x^{\prime}}{\partial x}\right)^{2}+\left(\frac{\partial y^{\prime}}{\partial x}\right)^{2}+\left(\frac{\partial z^{\prime}}{\partial x}\right)^{2}-\left(\frac{\partial t^{\prime}}{\partial x}\right)^{2}\right] . }
\end{aligned}
\end{gathered}
$$

We also have

$$
\begin{aligned}
& \frac{\partial\left(x^{\prime}, y^{\prime}, z^{\prime}, t^{\prime}\right)}{\partial(x, y, z, t)}= \frac{\partial\left(y^{\prime}, z^{\prime}\right)}{\partial(y, z)} \frac{\partial\left(x^{\prime}, t^{\prime}\right)}{\partial(x, t)}+\frac{\partial\left(z^{\prime}, x^{\prime}\right)}{\partial(y, z)} \frac{\partial\left(y^{\prime}, t^{\prime}\right)}{\partial(x, t)}+\ldots \\
&=\theta\left[\left\{\frac{\partial\left(x^{\prime}, t^{\prime}\right)}{\partial(x, t)}\right\}^{2}+\left\{\frac{\left.\partial\left(y^{\prime}, t^{\prime}\right)\right\}^{2}}{\partial(x, t)}\right\}^{2}+\ldots-\left\{\frac{\left.\partial\left(y^{\prime}, z^{\prime}\right)\right\}^{2}}{\partial(x, t)}\right\}^{2}-\ldots\right] \\
&=-\theta\left[\left\{\left(\frac{\partial x^{\prime}}{\partial x}\right)^{2}+\left(\frac{\partial y^{\prime}}{\partial x}\right)^{2}+\left(\frac{\partial z^{\prime}}{\partial x}\right)^{2}-\left(\frac{\partial t^{\prime}}{\partial x}\right)^{2}\right\}\right. \\
&\left.\times !\left(\frac{\partial x^{\prime}}{\partial t}\right)^{2}+\left(\frac{\partial y^{\prime}}{\partial t}\right)^{2}+\left(\frac{\partial z^{\prime}}{\partial t}\right)^{2}-\left(\frac{\partial t^{\prime}}{\partial t}\right)^{2}\right\} \\
&\left.-\left\{\frac{\partial x^{\prime}}{\partial x} \frac{\partial x^{\prime}}{\partial t}+\frac{\partial y^{\prime}}{\partial x} \frac{\partial y^{\prime}}{\partial t}+\frac{\partial z^{\prime}}{\partial x} \frac{\partial z^{\prime}}{\partial t}-\frac{\partial t^{\prime}}{\partial x} \frac{\partial t^{\prime} l^{2}}{\partial t}\right\}^{2}\right] .
\end{aligned}
$$

Therefore

$$
\begin{aligned}
\frac{\partial\left(x^{\prime}, y^{\prime}, z^{\prime}, t^{\prime}\right)}{\partial(x, y, z, t)}=-\theta\left[\left(\frac{\partial x^{\prime}}{\partial x}\right)^{2}+\left(\frac{\partial y^{\prime}}{\partial x}\right)^{2}\right. & \left.+\left(\frac{\partial z^{\prime}}{\partial x}\right)^{2}-\left(\frac{\partial t^{\prime}}{\partial x}\right)^{2}\right] \\
& \times\left[\left(\frac{\partial x^{\prime}}{\partial t}\right)^{2}+\left(\frac{\partial y^{\prime}}{\partial t}\right)^{2}+\left(\frac{\partial z^{\prime}}{\partial t}\right)^{2}-\left(\frac{\partial t^{\prime}}{\partial t}\right)^{2}\right] .
\end{aligned}
$$

This gives us the relation*

$$
-\frac{\partial\left(x^{\prime}, y^{\prime}, z^{\prime}\right)}{\partial(x, y, z)}\left[\left(\frac{\partial x^{\prime}}{\partial t}\right)^{2}+\left(\frac{\partial y^{\prime}}{\partial t}\right)^{2}+\left(\frac{\partial z^{\prime}}{\partial t}\right)^{2}-\left(\frac{\partial t^{\prime}}{\partial t}\right)^{2}\right]=\frac{\partial t^{\prime}}{\partial t} \frac{\partial\left(x^{\prime}, y^{\prime}, z^{\prime}, t^{\prime}\right)}{\partial(x, y, z, t)}
$$

which holds for any spherical wave transformation.

We shall now introduce the further restriction that the inequality

$$
\left(d x^{\prime}\right)^{2}+\left(d y^{\prime}\right)^{2}+\left(d z^{\prime}\right)^{2}<\left(d t^{\prime}\right)^{2}
$$

is a consequence of

$$
(d x)^{2}+(d y)^{2}+(d z)^{2}<(d t)^{2} .
$$

This means that, if a particle is moving with a velocity less than that of light in one system of coordinates, it is also moving with a velocity less than that of light in the transformed system.

* I am indebted to a referee for calling my attention to this relation and the nccessity of distinguishing between the two types of transformation. 
Putting

$$
(d x)^{2}+(d y)^{\prime}+\left(d z^{\prime}\right)^{2}-\left(d t^{\prime}\right)^{2}=\lambda^{2}\left[(d x)^{2}+(d y)^{2}+(d z)^{2}-(d t)^{2}\right],
$$

we see that $\lambda^{2}$ is positive, and therefore

$$
\left(\frac{\partial x^{\prime}}{\partial t}\right)^{2}+\left(\frac{\partial y^{\prime}}{\partial t}\right)^{2}+\left(\frac{\partial z^{\prime}}{\partial t}\right)^{2}-\left(\frac{\partial t^{\prime}}{\partial t}\right)^{2}
$$

is negative. This shows that $\frac{\partial\left(x^{\prime}, y^{\prime}, z^{\prime}\right)}{\partial(x, y, z)}$ and $\frac{\partial t^{\prime}}{\partial t} \frac{\partial\left(x^{\prime}, y^{\prime}, z^{\prime}, t^{\prime}\right)}{\partial(x, y, z, t)}$ must have the same sign. Hence, if $\frac{\partial t^{\prime}}{\partial t}$ is positive, $\frac{\partial\left(x^{\prime}, y^{\prime}, z^{\prime}\right)}{\partial(x, y, z)}$ must have the same sign as the Jacobian. Accordingly, a transformation which changes a right-handed system of axes into a right-handed system must have a positive Jacobian; a transformation which changes a right-handed system of axes into a left-handed system must have a negative Jacobian.

The sign of $\theta$ may now be determined from equation (A). Since

$$
\left(\frac{\partial x^{\prime}}{\partial x}\right)^{2}+\left(\frac{\partial y^{\prime}}{\partial x}\right)^{2}+\left(\frac{\partial z^{\prime}}{\partial x}\right)^{2}-\left(\frac{\partial t^{\prime}}{\partial x}\right)^{2}=\lambda^{2}
$$

it is necessarily positive. Consequently $\theta$ must have the same sign as $\frac{\partial\left(x^{\prime}, y^{\prime}, z^{\prime}\right)}{\partial(x, y, z)}$, and therefore the same sign as the Jacobian.

We can now obtain the formulæ of transformation in the two possible cases.

(i) When the Jacobian is positive, $\theta=+1$, and the formulæ of transformation are

$$
\begin{gathered}
E_{x}=E_{x}^{\prime} \frac{\partial\left(y^{\prime}, z^{\prime}\right)}{\partial(y, z)}+E_{y}^{\prime} \frac{\partial\left(z, x^{\prime}\right)}{\partial(y, z)}+E_{z}^{\prime} \frac{\partial\left(x^{\prime}, y^{\prime}\right)}{\partial(y, z)} \\
-H_{x}^{\prime} \frac{\partial\left(x^{\prime}, t^{\prime}\right)}{\partial(y, z)}-H_{y}^{\prime} \frac{\partial\left(y^{\prime}, t^{\prime}\right)}{\partial(y, z)}-H_{z}^{\prime} \frac{\partial\left(z^{\prime}, t^{\prime}\right)}{\partial(y, z)}, \\
-H_{x}=E_{x}^{\prime} \frac{\partial\left(y^{\prime}, z^{\prime}\right)}{\partial(x, t)}+E_{y}^{\prime} \frac{\partial\left(z^{\prime}, x^{\prime}\right)}{\partial(x, t)}+E_{z}^{\prime} \frac{\partial\left(x^{\prime}, y^{\prime}\right)}{\partial(x, t)} \\
-H_{x}^{\prime} \frac{\partial\left(x^{\prime}, t^{\prime}\right)}{\partial(x, t)}-H_{y}^{\prime} \frac{\partial\left(y^{\prime}, t^{\prime}\right)}{\partial(x, t)}-H_{z}^{\prime} \frac{\partial\left(z^{\prime}, t^{\prime}\right)}{\partial(x, t)}, \\
\rho w_{x}=\rho^{\prime} w_{x}^{\prime} \frac{\partial\left(y^{\prime}, z, t^{\prime}\right)}{\partial(y, z, t)}+\rho^{\prime} w_{y}^{\prime} \frac{\partial\left(z^{\prime}, x^{\prime}, t^{\prime}\right)}{\partial(y, z, t)}+\rho^{\prime} w_{z}^{\prime} \frac{\partial\left(x^{\prime}, y^{\prime}, t^{\prime}\right)}{\partial(y, z, t)}-\rho^{\prime} \frac{\partial\left(x^{\prime}, y^{\prime}, z^{\prime}\right)}{\partial(y, z, t)}, \\
-\rho=\rho^{\prime} w_{x}^{\prime} \frac{\partial\left(y^{\prime}, z^{\prime}, t^{\prime}\right)}{\partial(x, y, z)}+\rho^{\prime} w_{y}^{\prime} \frac{\partial\left(z^{\prime}, x^{\prime}, t^{\prime}\right)}{\partial(x, y, z)}+\rho^{\prime} w_{z}^{\prime} \frac{\partial\left(x^{\prime}, y^{\prime}, t^{\prime}\right)}{\partial(x, y, z)}-\rho^{\prime} \frac{\partial\left(x^{\prime}, y, z^{\prime}\right)}{\partial(x, y, z)}
\end{gathered}
$$


These equations may be simplified by using the relations of type

$$
\frac{\partial\left(y, z, t^{\prime}\right)}{\partial(x, y, z)}=\theta \frac{\partial x^{\prime}}{\partial t} \lambda^{2}
$$

which are proved in the same way as (A), the quantity $\lambda^{2}$ being defined by equation (C).

The new equations are

$$
\begin{aligned}
& \rho w_{x}=\lambda^{2}\left[\rho^{\prime} w_{x}^{\prime} \frac{\partial x^{\prime}}{\partial x}+\rho^{\prime} w_{y}^{\prime} \frac{\partial y}{\partial x}+\rho^{\prime} w_{z}^{\prime} \frac{\partial z^{\prime}}{\partial x}-\rho^{\prime} \frac{\partial t^{\prime}}{\partial x}\right], \\
& -\rho=\lambda^{2}\left[\rho^{\prime} w_{x}^{\prime} \frac{\partial x^{\prime}}{\partial t}+\rho^{\prime} w_{y}^{\prime} \frac{\partial y^{\prime}}{\partial t}+\rho^{\prime} w_{z}^{\prime} \frac{\partial z^{\prime}}{\partial t}-\rho^{\prime} \frac{\partial t^{\prime}}{\partial t}\right],
\end{aligned}
$$

and these imply that

$$
\rho w_{x} d x+\rho w_{y} d y+\rho w_{z} d z-\rho d t=\lambda^{2}\left[\rho^{\prime} w_{x}^{\prime} d x^{\prime}+\rho^{\prime} w_{y}^{\prime} d y^{\prime}+\rho^{\prime} w_{z}^{\prime} d z^{\prime}-\rho^{\prime} d t^{\prime}\right] .
$$

The formulæ connecting the electromagnetic potentials are obtained by putting

$$
A_{x} d x+A_{y} d y+A_{z} d z-\Phi d t=\theta\left[A_{x}^{\prime} d x^{\prime}+A_{y}^{\prime} d y^{\prime}+A_{z}^{\prime} d z^{\prime}-\Phi^{\prime} d \cdot t^{\prime}\right] .
$$

Since $\theta=+1$, they are

$$
\begin{gathered}
A_{x}=A_{x}^{\prime} \frac{\partial x^{\prime}}{\partial x}+A_{y}^{\prime} \frac{\partial y^{\prime}}{\partial x}+A_{z}^{\prime} \frac{\partial z}{\partial x}-\Phi^{\prime} \frac{\partial t}{\partial x} \\
-\Phi=A_{x}^{\prime} \frac{\partial x^{\prime}}{\partial t}+A_{y}^{\prime} \frac{\partial y^{\prime}}{\partial t}+A_{z}^{\prime} \frac{\partial z^{\prime}}{\partial t}-\Phi^{\prime} \frac{\partial t^{\prime}}{\partial t}
\end{gathered}
$$

(ii) When the Jacobian is negative and $\partial t^{\prime} / \partial t>0$, the axes are righthanded in the original system and left-handed in the system specified by the dashed letters. To obtain the correct formulæ of transformation we must change the sign of $H^{\prime}$ in equations (2) and (3) and put $\theta=-1$. There is also a doubt about the sign in the equation

$\rho w d y d z d t+\rho w_{y} d z d x d t+\rho w_{z} d x d y d t-\rho d x d y d z$

$$
= \pm\left(\rho^{\prime} w_{x} d y^{\prime} d z^{\prime} d t^{\prime}+\rho w_{y} d z^{\prime} d x^{\prime} d t^{\prime}+\rho^{\prime} w_{z} d x^{\prime} d y^{\prime} d t^{\prime}-\rho^{\prime} d x^{\prime} d y^{\prime} d x^{\prime}\right),
$$

the sign depending upon whether positive electricity is transformed into negative electricity or positive electricity. If the negative sign be taken there must be a corresponding alteration in equation (3). 
4. Spherical Wave Transformations and the Group of Conformal Transformations of a Space of Four Dimensions.

The group of spherical wave transformations may be reduced to a known group by putting $t=i s$. The quadratic form which remains invariant is then of the type

$$
\lambda^{2}\left(d x^{2}+d y^{2}+d z^{2}+d s^{2}\right),
$$

and so the transformation is a conformal one.

The group of conformal transformations in a space of four dimensions has been studied by Sophus Lie, ${ }^{*}$ who has shown that it is composed of reflexions, translations, rotations, magnifications, and inversions. $t$ The transformations which are of importance in the present case are the imaginary ones, and it should be noticed that by a combination of two imaginary inversions we can obtain a transformation of the type

$$
\begin{aligned}
x^{\prime}=\frac{k x}{z+i s}, \quad y^{\prime} & =\frac{k y}{z+i s}, \quad z^{\prime}=k \frac{x^{2}+y^{2}+z^{2}+s^{2}-a^{2}}{2 a(z+i s)}, \\
s^{\prime} & =k \frac{x^{2}+y^{2}+z^{2}+s^{2}+a^{2}}{2 i a(z+i s)},
\end{aligned}
$$

which is quite different from an inversion or simple displacement. This corresponds to the real spherical wave transformation $\ddagger$

$$
\begin{aligned}
x^{\prime}=\frac{k x}{z-t}, \quad y^{\prime} & =\frac{k y}{z-t}, \quad z^{\prime}=k \frac{x^{2}+y^{2}+z^{2}-a^{2}-t^{2}}{2 a(z-t)}, \\
-t^{\prime} & =k \frac{x^{2}+y^{2}+z^{2}+a^{2}-t^{2}}{2 a(z-t)} .
\end{aligned}
$$

An imaginary rotation in the four-dimensional space may be specified, in a particular case, by the equations

$$
\begin{array}{ll}
x^{\prime}=x \cos i w+s \sin i w, & y^{\prime}=y, \\
s^{\prime}=-x \sin i w+s \cos i w, & z^{\prime}=z .
\end{array}
$$

Putting $\tanh w=v$,

* Göttinger Nachrichten (1871), Transformationgruppen, Bd. 3, p. 351.

$\dagger$ Every transformation belonging to the group is a birational transformation.

$\ddagger$ See a paper by the author "On the Conformal Transformations of a Space of Four Dimensions and their Applications to Geometrical Optics," Proc. London Math. Soc., Ser. 2, Vol. 7, p. 70 (1909). 
we obtain Lorentz's transformation

$$
x^{\prime}=\frac{x+v t}{\sqrt{ }\left(1-v^{2}\right)}, \quad y^{\prime}=y, \quad z^{\prime}=z, \quad t^{\prime}=\frac{t+v x}{\sqrt{ }\left(1-v^{2}\right)} .
$$

The formulæ of transformation of the electric and magnetic vectors are obtained at once from the general formulæ; they are

$$
\begin{array}{cl}
A_{x}=\beta\left(A_{x}^{\prime}-v \Phi^{\prime}\right), & -\Phi=\beta\left(v A_{x}^{\prime}-\Phi^{\prime}\right), \\
A_{y}=A_{y}^{\prime}, & A_{z}=A_{z}^{\prime}, \\
E_{x}=E_{x}^{\prime}, & H_{x}=H_{x}^{\prime}, \\
E_{y}=\beta\left(E_{y}^{\prime}-v H_{z}^{\prime}\right), & H_{y}=\beta\left(H_{y}^{\prime}+v E_{z}^{\prime}\right), \\
E_{z}=\beta\left(E_{z}^{\prime}+v H_{y}^{\prime}\right), & H_{z}=\beta\left(H_{z}^{\prime}-v E_{y}^{\prime}\right), \\
\rho w_{x}=\beta\left(\rho^{\prime} w_{x}^{\prime}-v \rho^{\prime}\right), \quad \rho w_{y}=\rho^{\prime} w_{y}^{\prime}, \quad \rho w_{z}=\rho^{\prime} w_{z}^{\prime}, \\
-\rho=\beta\left(v \rho^{\prime} w_{x}^{\prime}-\rho^{\prime}\right),
\end{array}
$$

where

$$
\beta=\frac{1}{\sqrt{ }\left(1-v^{2}\right)} .
$$

These agree with the formulæ of Einstein, Lorentz's formulæ for the convection currents being slightly different.

In the case of an inversion with regard to a hypersphere whose centre is at the origin, and whose radius is a real quantity $k$, the formulæ of transformation are

$$
\begin{aligned}
x^{\prime}=\frac{k^{2} x}{x^{2}+y^{2}+z^{2}+s^{2}}, \quad y^{\prime} & =\frac{k^{2} y}{x^{2}+y^{2}+z^{2}+s^{2}}, \quad z^{\prime}=\frac{k^{2} z}{x^{2}+y^{2}+z^{2}+s^{2}}, \\
s^{\prime} & =\frac{k^{2} s}{x^{2}+y^{2}+z^{2}+s^{2}},
\end{aligned}
$$

Putting $s=i t, x^{2}+y^{2}+z^{2}=r^{2}$, we get the real spherical wave transformation

$$
x^{\prime}=\frac{k^{2} x}{r^{2}-t^{2}}, \quad y^{\prime}=\frac{k^{2} y}{r^{2}-t^{2}}, \quad z^{\prime}=\frac{k^{2} z}{r^{2}-t^{2}}, \quad t^{\prime}=\frac{k^{2} t}{r^{2}-t^{2}} .
$$

This transformation has a negative Jacobian; the formulæ for transforming the components of the electric and magnetic force have been obtained by E. Cunningham.*

It should be noticed that

$$
\frac{d t^{\prime}}{d t}=\frac{r^{2}+t^{2}}{\left(r^{2}-t^{2}\right)^{2}}
$$

* Proc. London Math. Soc., Ser, 2, Vol. 8, p. 77.

SIIR. 2. VOL. 8. NO. 1051. 
and is positive; hence $t^{\prime}$ increases as $t$ increases, if $(x, y, z)$ are kept constant.

The transformation which corresponds to a reflexion in the fourdimensional space is also of considerable interest. In the particular case when the reflecting space passes through the plane $x=0, s=0$, the reflexion may be replaced by a rotation round the plane $x=0, s=0$, and a reflexion in the space $x=0$. The corresponding transformation is thus made up out of a transformation of Lorentz

$$
x^{\prime}=\frac{x-v t}{\sqrt{ }\left(1-v^{2}\right)}, \quad y^{\prime}=y, \quad z^{\prime}=z, \quad t^{\prime}=\frac{t-v x}{\sqrt{ }\left(1-v^{2}\right)},
$$

and a change in the sign of $x^{\prime}$. Putting

$$
\frac{v}{\sqrt{ }\left(1-v^{2}\right)}=\frac{2 u}{1-u^{2}}, \quad \frac{1}{\sqrt{ }\left(1-v^{2}\right)}=\frac{1+u^{2}}{1-u^{2}},
$$

and changing the sign of $x^{\prime}$, we get

$$
\begin{aligned}
x^{\prime} & =-\frac{1+u^{2}}{1-u^{2}} x+\frac{2 u}{1-u^{2}} t, \\
y^{\prime} & =y, \\
z^{\prime} & =z, \\
t^{\prime} & =\frac{1+u^{2}}{1-u^{2}} t-\frac{2 u}{1-u^{2}} x .
\end{aligned}
$$

The quantity $u$ is introduced because the angle of rotation in the fourdimensional space is twice the angle between the reflecting space and the space $x=0$. Its geometrical meaning in the case of the spherical wave transformation is indicated by the equation

$$
x^{\prime}-u t^{\prime}=-(x-u t),
$$

which implies that a plane moving with the constant velocity $u$ is transformed into itself. Further, when $x=u t$, we have

$$
x^{\prime}=x, \quad y^{\prime}=y, \quad z^{\prime}=z, \quad t^{\prime}=t ;
$$

hence every point of the plane is transformed into itself. ${ }^{*}$

The formulæ of transformation of the electromagnetic vectors are found from the first set of equations for a transformation with negative Jacobian.

* Geometrically the transformation is equivalent to a reflexion in a moving plane mirror. 
T he general infinitesimal spherical wave transformation is

$$
\begin{aligned}
& x^{\prime}=x+\epsilon\left[p\left(x^{2}-y^{2}-z^{2}+t^{2}\right)+2 q x y+2 r x z+2 s x t+\mu x+h y+g z+l t+a\right], \\
& y^{\prime}=y+\epsilon\left[q\left(y^{2}-z^{2}-x^{2}+t^{2}\right)+2 p x y+2 r y z+2 s y t-h x+\mu y+f z+m t+b\right], \\
& z^{\prime}=z+\epsilon\left[r\left(z^{2}-x^{2}-y^{2}+t^{2}\right)+2 p x z+2 q y z+2 s z t-g x-f y+\mu z+n t+c\right], \\
& t^{\prime}=t+\epsilon\left[s\left(x^{2}+y^{2}+z^{2}+t^{2}\right)+2 p x t+2 q y t+2 r z t+l x+m y+n z+\mu t+d\right],
\end{aligned}
$$

where the coefficients are all constants, and $\epsilon$ is a quantity whose square may be neglected. Since there are fifteen arbitrary constants the group is a fifteen parameter group.

We have

$$
\begin{gathered}
\frac{\partial x^{\prime}}{\partial x}=\frac{\partial y^{\prime}}{\partial y}=\frac{\partial z^{\prime}}{\partial z}=\frac{\partial t^{\prime}}{\partial t}=1+\epsilon[\mu+2 p x+2 q y+2 r z+2 s t]=\lambda, \\
d t^{\prime 2}-d x^{\prime 2}-d y^{\prime 2}-d z^{\prime 2}=\lambda^{2}\left[d t^{2}-d x^{2}-d y^{2}-d z^{2}\right] .
\end{gathered}
$$

If $(x, y, z)$ is kept constant as $t$ varies, the corresponding point $\left(x^{\prime}, y^{\prime}, z^{\prime}\right)$ moves along a parabola, but in one type of transformation it moves along a straight line with constant acceleration. This is the case, for example, when

$$
\begin{aligned}
x^{\prime} & =x+\epsilon\left(y^{2}+z^{2}-x^{2}-t^{2}\right), & y^{\prime} & =y(1-2 \epsilon x), \\
z^{\prime} & =z(1-2 \epsilon x), & t^{\prime} & =t(1-2 \epsilon x),
\end{aligned}
$$

for, since quantities of order $\epsilon^{2}$ may be neglected, the first equation may be written

$$
x^{\prime}=x+\epsilon\left(y^{2}+z^{2}-x^{2}-t^{\prime 2}\right) \text {. }
$$

Hence, if $(x, y, z)$ are kept constant, the point $\left(x^{\prime}, y^{\prime}, z^{\prime}\right)$ moves with constant acceleration $\gamma$ given by

$$
\gamma=-2 \epsilon
$$

Substituting for $\gamma$, we get

$$
\begin{aligned}
x^{\prime} & =x-\frac{1}{2} \gamma\left(y^{2}+z^{2}-x^{2}-t^{2}\right), & & y^{\prime}=y(1+\gamma x), \\
z^{\prime} & =z(1+\gamma x), & t^{\prime} & =t(1+\gamma x) .
\end{aligned}
$$

The last equation agrees with the one obtained by Einstein.*

In the case of the general infinitesimal transformation, the expression

$$
\left(x^{\prime}-x\right)^{2}+\left(y^{\prime}-y\right)^{2}+\left(z^{\prime}-z\right)^{2}-\left(t^{\prime}-t\right)^{2},
$$


is of order $\epsilon^{2}$, but there is one type of transformation in which it is of order $\epsilon^{3}$ at least. The formulæ of transformation are then

$$
\begin{aligned}
& x^{\prime}=x+\epsilon\left[2 x(l x+m y+n z-t)-l\left(x^{2}+y^{2}+z^{2}-t^{2}\right)\right], \\
& y^{\prime}=y+\epsilon\left[2 y(l x+m y+n z-t)-m\left(x^{2}+y^{2}+z^{2}-t^{2}\right)\right], \\
& z^{\prime}=z+\epsilon\left[2 z(l x+m y+n z-t)-n\left(x^{2}+y^{2}+z^{2}-t^{2}\right)\right], \\
& t^{\prime}=t+\epsilon\left[2 t(l x+m y+n z-t)-\left(x^{2}+y^{2}+z^{2}-t^{2}\right)\right],
\end{aligned}
$$

where

$$
l^{2}+n^{2}+n^{2}=1,
$$

and signify that $\left(x^{\prime}, y^{\prime}, z^{\prime}\right),(x, y, z)$ are successive positions of a particle which is moving with the velocity of light.

Real spherical wave transformations may be obtained geometrically in the following way. Let the space time point $(x, y, z, t)$ be represented by a sphere of radius $t$ having its centre at the point $(x, y, z)$. Then, if we apply a real conformal transformation of space to these representative spheres, the new set of spheres may be taken as the representative spheres of a new set of space time points $\left(x^{\prime}, y^{\prime}, z^{\prime}, t^{\prime}\right)$. These are connected with the original set by a system of equations which define a spherical wave transformation. This theorem has already been established in the case of an inversion, ${ }^{*}$ and since any real conformal transformation of space can be built up from inversions, it follows that the general transformation obtained in the above way is a spherical wave transformation.

It should be noticed that the general spherical wave transformation cannot be obtained in this way, because $t^{\prime}=0$ always corresponds to $t=0$. If, however, we combine these transformations with the real spherical wave transformation obtained by increasing or decreasing the radii of all the representative spheres by the same amount, it is possible to obtain any spherical wave transformation by a suitable combination. The proof of this will be left to the reader.

When we use the representative spheres the differential equation

$$
(d x)^{2}+(d y)^{2}+(d z)^{2}-(d t)^{2}=0,
$$

admits of a very simple interpretation, as it implies that the two consecutive representative spheres specified by $(x, y, z, t)$ and $(x+d x, y+d y$, $z+d z, t+d t)$ touch one another internally. 


\section{The Transformation of Integral Forms.}

The general theory of the transformation of physical problems by means of a change of coordinates can be developed in a convenient manner by studying a number of integral forms, examining the way in which they are related to one another, and obtaining the formulæ by means of which they can be transformed.*

We shall commence by studying the simple case of a transformation in two variables from $(x, y)$ to $\left(x^{\prime}, y^{\prime}\right)$. Suppose that

$$
\begin{aligned}
a d x+b d y & =a^{\prime} d x^{\prime}+b^{\prime} d y^{\prime}, \\
A d x+B d y & =A^{\prime} d x^{\prime}+B^{\prime} d y^{\prime},
\end{aligned}
$$

then

$$
\begin{array}{ll}
a=a^{\prime} \frac{\partial x^{\prime}}{\partial x}+b^{\prime} \frac{\partial y^{\prime}}{\partial x}, & b=a^{\prime} \frac{\partial x^{\prime}}{\partial y}+b^{\prime} \frac{\partial y^{\prime}}{\partial y}, \\
A=A^{\prime} \frac{\partial x^{\prime}}{\partial x}+B^{\prime} \frac{\partial y^{\prime}}{\partial x}, & B=A^{\prime} \frac{\partial x^{\prime}}{\partial y}+B^{\prime} \frac{\partial y^{\prime}}{\partial y},
\end{array}
$$

and it is easy to see that

$$
a B-A b=\left(a^{\prime} B^{\prime}-A^{\prime} b^{\prime}\right) \frac{\partial\left(x^{\prime}, y^{\prime}\right)}{\partial(x, y)} .
$$

This implies that $(a B-A b) d x d y=\left(a^{\prime} B^{\prime}-A^{\prime} b^{\prime}\right) d x^{\prime} d y^{\prime}$.

Now this relation can be obtained from the previous pair by the process of multiplication used in Grassmann's calculus of extension. $t$ In this calculus, the sign of a product depends on the order of the terms; thus

$$
d x d y=-d y d x \text { and } d x d x=0 .
$$

This rule can also be applied to the case in which the quantities $a$ and $b$ are differential operators; thus, if

$$
\begin{aligned}
& a=\frac{\partial}{\hat{c} x}, \quad b=\frac{\partial}{\partial y}, \quad a^{\prime}=\frac{\hat{c}}{\partial x^{\prime}}, \quad b^{\prime}=\frac{\hat{c}}{\partial y^{\prime}}, \\
& \left(\frac{\partial B}{\partial x}-\frac{\partial A}{\partial y}\right) d x d y=\left(\frac{\hat{\partial} B^{\prime}}{\partial x^{\prime}}-\frac{\partial A^{\prime}}{\partial y^{\prime}}\right) d x^{\prime} d y^{\prime},
\end{aligned}
$$

a relation which may be obtained directly by means of Green's theorem.

* The theory of integral invariants has been developed by Poincaré, Mécanique céleste, t. 3 ; Methodes nouvelles de la Mécanique céleste, t. 3, p. 33 ; Goursat, Liouville's Journal (6), t. 4, p. 331 ; Koenigs, Comptes rendus, t. 122 (1906), pp. 25-27.

† Scott's Determinants, p. 16. 
Next, suppose that the formula of transformation of a quadratic differential form is known, e.g.,

$$
E d x^{2}+2 F d x d y+G d y^{2}=E^{\prime} d x^{\prime 2}+2 F^{\prime} d x^{\prime} d y^{\prime}+G^{\prime} d y^{\prime 2} ;
$$

then taking two independent differentials $d x, d y, \delta x, \delta y$, and writing $d x+\lambda \delta x, d y+\lambda \delta y, \ldots$ in place of $d x, d y$, we get the formula of transformation of the bilinear form

$E d x \delta x+F(d x \delta y+d y \delta x)+G d y \delta y$

$$
=E^{\prime} d x^{\prime} \delta x^{\prime}+F^{\prime}\left(d x^{\prime} \delta y^{\prime}+d y^{\prime} \delta x^{\prime}\right)+G^{\prime} d y^{\prime} \delta y^{\prime} .
$$

We may multiply this equation by itself, multiplying both sets of differentials according to Grassmann's rule. The resulting equation is

$$
\left(E G-F^{2}\right) d x d y \delta x \delta y=\left(E^{\prime} G^{\prime}-F^{\prime 2}\right) d x^{\prime} d y^{\prime} \delta x^{\prime} \delta y^{\prime} .
$$

This gives

$$
\sqrt{ }\left(E G-F^{2}\right) d x d y=\sqrt{ }\left(E^{\prime} G^{\prime}-F^{\prime 2}\right) d x^{\prime} d \cdot y^{\prime}
$$

or

$$
\Delta d x d y=\Delta^{\prime} d x^{\prime} d y^{\prime} \text {. }
$$

Multiplying (6) by (1) according to Grassmann's rule, we obtain

$$
\begin{aligned}
& {[(E b-F a) \delta x-(G a-F b) \delta y] d x d y} \\
& =\left[\left(E^{\prime} b^{\prime}-F^{\prime \prime} a^{\prime}\right) \delta x^{\prime}-\left(G^{\prime} a^{\prime}-F^{\prime \prime} b^{\prime}\right) \delta y^{\prime}\right] d x^{\prime} d y^{\prime} .
\end{aligned}
$$

This gives the formula of transformation of a linear form

$$
\frac{E b-F a}{\Delta} \delta x-\frac{G a-F b}{\Delta} \delta y=\frac{E^{\prime} b^{\prime}-F^{\prime} a^{\prime}}{\Delta} \delta x^{\prime}-\frac{G^{\prime} a^{\prime}-F^{\prime} b^{\prime}}{\Delta^{\prime}} \delta y^{\prime},
$$

which may be called the reciprocal of the first.

Multiplying this equation by

respectively, we get

$$
\begin{gathered}
a d x+b d y=a^{\prime} d x^{\prime}+b^{\prime} d y^{\prime}, \\
d x \frac{\partial}{\partial x}+d y \frac{\partial}{\partial y}=d x^{\prime} \frac{\partial}{\partial x}+d y^{\prime} \frac{\partial}{\partial y^{\prime}},
\end{gathered}
$$

$$
\begin{gathered}
\frac{E b^{2}-2 F a b+G a^{2}}{\Delta} d x d y=\frac{E^{\prime} b^{\prime 2}-2 F^{\prime} a^{\prime} b^{\prime}+G^{\prime} a^{\prime 2}}{\Delta^{\prime}} d x^{\prime} d y \\
{\left[\frac{\partial}{\partial x}\left(\frac{G a-F b}{\Delta}\right)+\frac{\partial}{\partial y}\left(\frac{E b-F a}{\Delta}\right)\right] d x d y} \\
=\left[\frac{\partial}{\partial x^{\prime}}\left(\frac{G^{\prime} a^{\prime}-F^{\prime} b^{\prime}}{\Delta^{\prime}}\right)+\frac{\partial}{\partial y^{\prime}}\left(\frac{E^{\prime} b^{\prime}-F^{\prime} a^{\prime}}{\Delta^{\prime}}\right)\right] d x^{\prime} d y^{\prime}
\end{gathered}
$$


These formulæ are well known in the differential geometry of a sur. face.*

We shall now show that the above theory may be extended to transformations in any number of variables. In the first place we must show that the law of multiplication still holds. Let there be $n$ variables $x_{1}, \ldots, x_{n}$, and suppose that

$$
\begin{aligned}
& \Sigma a_{r} d x_{r}=\Sigma a_{r}^{\prime} d x_{r}^{\prime}, \\
& \Sigma b_{r} d x_{r}=\Sigma b_{r}^{\prime} d x_{r}^{\prime} ;
\end{aligned}
$$

then by multiplication we may obtain an integral form of the second order $\dagger$

$$
\Sigma\left(a_{r} b_{s}-a_{s} b_{r}\right) d x_{r} d x_{s}=\Sigma\left(a_{r}^{\prime} b_{s}^{\prime}-a_{s}^{\prime} b_{r}^{\prime}\right) d x_{r}^{\prime} d x_{s}^{\prime},
$$

the multiplication being performed by Grassmann's rule. To verify this we have only to replace $d x_{r} d x_{s}$ by $\frac{\partial\left(x_{r}, x_{s}\right)}{\partial(\alpha, \beta)} d \alpha d \beta$, and notice that the last equation may be written

$$
\left|\begin{array}{ll}
\Sigma a_{r} \frac{\partial x_{r}}{\partial \alpha}, & \Sigma b_{r} \frac{\partial x_{r}}{\partial a} \\
\Sigma a_{r} \frac{\partial x_{r}}{\partial \beta}, & \Sigma b_{r} \frac{\partial x_{r}}{\partial \beta}
\end{array}\right| d a d \beta=\left|\begin{array}{cc}
\Sigma a_{r}^{\prime} \frac{\partial x_{r}^{\prime}}{\partial a}, & \Sigma b_{r}^{\prime} \frac{\partial x_{r}^{\prime}}{\partial \alpha} \\
\Sigma a_{r}^{\prime} \frac{\partial x_{r}^{\prime}}{\partial \beta}, & \Sigma b_{r}^{\prime} \frac{\partial x_{r}^{\prime}}{\partial \beta}
\end{array}\right| d \alpha d \beta .
$$

Similarly, if we take three integral forms,

$$
\begin{aligned}
& \Sigma a_{r} d x_{r}=\Sigma a_{r}^{\prime} d x_{r}^{\prime}, \\
& \Sigma b_{r} d x_{r}=\Sigma b_{r}^{\prime} d x_{r}^{\prime}, \\
& \Sigma c_{r} d x_{r}=\Sigma c_{r_{r}}^{\prime} d x_{r}^{\prime},
\end{aligned}
$$

and multiply them together by Grassmann's rule, we obtain the integral

* Darboux, 'thèorie générale des Surfaces, t. 3, p. 193.

In particular, if

$$
d x^{2}+d y^{2}=\lambda\left[d x^{\prime 2}+d y^{\prime 2}\right],
$$

we may deduce from the identity

that

$$
\begin{aligned}
& \frac{\partial V}{\partial x} d x+\frac{\partial V}{\partial y} d y=\frac{\partial V}{\partial x^{\prime}} d x^{\prime}+\frac{\partial V}{\partial y^{\prime}} d y^{\prime}, \\
& \partial V d x-\frac{\partial V}{\partial x} d y=\frac{\partial V}{\partial y^{\prime}} d x^{\prime}-\frac{\partial V}{\partial x^{\prime}} d y^{\prime},
\end{aligned}
$$

and on multiplying by (9), we get the well known relation

$$
\left(\begin{array}{l}
\partial^{2} V \\
\partial x^{2}
\end{array}+\frac{\partial^{3} V}{\partial y^{i}}\right) d x d y=\left(\frac{\partial^{2} V}{\partial x^{\prime 2}}+\frac{\partial^{2} V}{\partial y^{\prime i}}\right) d x^{\prime} d y^{\prime} .
$$

$\dagger$ An integral form of the second order must be carefully distinguished from it quadratic differential form. 
form of the third order

$$
\sum_{r, s, t}\left|\begin{array}{ccc}
a_{r} & a_{s} & a_{t} \\
b_{r} & b_{s} & b_{t} \\
c_{r} & c_{s} & c_{t}
\end{array}\right| d x_{r} d x_{s} d x_{t}=\sum_{r, s, t}\left|\begin{array}{ccc}
a_{r}^{\prime} & a_{s}^{\prime} & a_{t}^{\prime} \\
b_{r}^{\prime} & b_{s}^{\prime} & b_{t}^{\prime} \\
c_{r}^{\prime} & c_{s}^{\prime} & c_{t}^{\prime}
\end{array}\right| d x_{r}^{\prime} d x_{s}^{\prime} d x_{t}^{\prime}
$$

It will be seen also that this equation may be obtained by multiplying the integral form of the second order derived from $(\alpha)$ and $(\beta)$, by $(\gamma)$. The question then arises whether integral forms of any order may be multiplied together by Grassmann's rule. To show that this is the case we shall consider the two integral forms

$$
\begin{aligned}
& \sum_{s, t, \ldots} A_{s, t} \ldots d x_{s} d x_{t} \ldots=\sum_{s, t, \ldots} A_{s, t, \ldots}^{\prime} d x_{s}^{\prime} d x_{t}^{\prime}, \ldots, \\
& \sum_{p, y, r, \ldots} B_{p, \psi_{1}, r, \ldots} d x_{p} d x_{q} d x_{r}=\sum_{p, y, r, \ldots} B_{p, y, r, \ldots}^{\prime} d x_{p}^{\prime} d x_{1}^{\prime} d x_{r}^{\prime} \ldots,
\end{aligned}
$$

of orders $k$ and $m$ respectively. Choosing $m+k$ variables $a, \beta, \ldots$, we have $\frac{(m+k) !}{m ! k !}$ relations of the type

$$
\sum_{s, t, \ldots} A_{s, t} \ldots \frac{\partial\left(x_{s}, x_{t}, \ldots\right)}{\partial(\alpha, \beta, \ldots)}=\sum_{s, t, \ldots} A_{s, t, \ldots}^{\prime} \frac{\partial\left(x_{s}^{\prime}, x_{t}^{\prime}, \ldots\right)}{\partial(\alpha, \beta, \ldots)},
$$

and the same number of relations of the type

$$
\sum_{p, \psi_{,}, \ldots, \ldots} B_{p, \psi, r, \ldots} \frac{\partial\left(x_{p}, x_{\eta,}, x_{r}, \ldots\right)}{\partial(\gamma, \delta, \epsilon, \ldots)}=\sum_{p, q, r, \ldots} B_{p, q, r, \ldots}^{\prime} \frac{\partial\left(x_{p}^{\prime}, x_{\eta}^{\prime}, x_{r}^{\prime}, \ldots\right)}{\partial(\gamma, \delta, \epsilon, \ldots)} .
$$

These may be arranged in conjugate pairs in such a way that the whole set of variables $\alpha, \beta, \gamma, \delta, \ldots$ occur in each pair. Multiplying conjugate pairs together, attributing proper signs to each product, and adding, we find from the properties of the minors of a determinant that the coefficient of a term such as $A_{s, t} \ldots B_{p, u, r, \ldots}$ is zero, unless the quantities $s, t, p, q, r, \ldots$ are all different. When these quantities are different the coefficient is simply the determinant $\frac{\partial\left(x_{s}, x_{t}, \ldots, x_{p}, x_{q}, x_{r}, \ldots\right)}{\partial(\alpha, \beta, \gamma, \ldots)}$ with a proper sign. When multiplied by $d \alpha d \beta d \gamma \ldots$ this yields $d x_{s} d x_{t} \ldots d x_{p} d x_{1} d x_{r} \ldots$, and it is clear from this that the multiplication of the integral forms by Grassmann's rule is justified.

Taking the case of a transformation from four variables $(x, y, z, t)$ to $\left(x^{\prime}, y^{\prime}, z^{\prime}, t^{\prime}\right)$. If

$H_{x} d y d z+H_{y} d z d x+H_{z} d x d y+E_{x} d x d t+E_{y} d y d t+E_{z} d z d t$

$$
=H_{x}^{\prime} d y^{\prime} d z^{\prime}+H_{y}^{\prime} d z^{\prime} d x^{\prime}+H_{z}^{\prime} d x^{\prime} d y^{\prime}+E_{x}^{\prime} d x^{\prime} d t^{\prime}+E_{y}^{\prime} d y^{\prime} d t^{\prime}+E_{x}^{\prime} d z^{\prime} d t^{\prime},
$$


and

$E_{x} d y d z+E_{y} d z d x+E_{z} d x d y-H_{x} d x d t-H_{y} d y d t-H_{z} d z d t$

$$
=E_{x}^{\prime} d y^{\prime} d z^{\prime}+E_{y}^{\prime} d z^{\prime} d x^{\prime}+E_{z}^{\prime} d x^{\prime} d y^{\prime}-H_{x} d x^{\prime} d t^{\prime}-H_{y}^{\prime} d y^{\prime} d t^{\prime}-H_{z}^{\prime} d z^{\prime} d t^{\prime},
$$

we obtain, on multiplication,

$$
\begin{aligned}
\left(E_{x}^{2}+E_{y}^{2}+E_{z}^{2}-H_{x}^{2}-H_{y}^{2}-H_{z}^{2}\right) d x d y d z d t \\
=\left(E_{x}^{\prime 2}+E_{y}^{\prime 2}+E_{z}^{\prime 2}-H_{x}^{\prime 2}-H_{y}^{\prime 2}-H_{z}^{\prime 2}\right) d x^{\prime} d y^{\prime} d z^{\prime} d t^{\prime},
\end{aligned}
$$

while, if eithei form be multiplied by itself, we obtain

$$
\left(E_{x} H_{x}+E_{y} H_{y}+E_{z} H_{z}\right) d x d y d z d t=\left(E_{y}^{\prime} H_{x}^{\prime}+E_{y}^{\prime} H_{y}^{\prime}+E_{z}^{\prime} H_{z}^{\prime}\right) d x^{\prime} d y^{\prime} d z^{\prime} d t^{\prime} .
$$

These equations indicate the invariance of the property that at a surface of discontinuity, or in a spherical wave, the electric force is equal in magnitude to the magnetic force and perpendicular to it.

A space time transformation from the variables $(x, y, z, t)$ to $\left(x^{\prime}, y^{\prime}, z^{\prime}, t^{\prime}\right)$ can be used to transform the whole motion in one dynamical system into a corresponding motion in another, as far as the kinematics is concerned, provided the velocities $\left(w_{x}, w_{y}, w_{z}\right),\left(w_{x}^{\prime}, w_{y}^{\prime}, w_{z}^{\prime}\right)$ of corresponding points are such that the equations

$$
d x^{\prime}=w_{x}^{\prime} d t^{\prime}, \quad d y^{\prime}=w_{y}^{\prime} d t^{\prime}, \quad d z^{\prime}=w_{z}^{\prime} d t^{\prime},
$$

are a consequence of the relations

$$
d x=w_{x} d t, \quad d y=w_{y} d t, \quad d z=v_{z} d t .
$$

This condition is satisfied, if

$$
\begin{aligned}
& d x^{\prime}-w_{x}^{\prime} d t^{\prime}=\nu_{11}\left(d x-w_{x} d t\right)+\nu_{12}\left(d y-w_{y} d t\right)+\nu_{18}\left(d z-w_{z} d t\right), \\
& d y^{\prime}-w_{y}^{\prime} d t^{\prime}=\nu_{21}\left(d x-w_{x} d t\right)+\nu_{22}\left(d y-w_{y} d t\right)+\nu_{23}\left(d z-w_{z} d t\right), \\
& d z^{\prime}-w_{z}^{\prime} d t^{\prime}=\nu_{31}\left(d x-w_{x} d t\right)+\nu_{32}\left(d y-w_{y} d t\right)+\nu_{33}\left(d z-w_{z} d t\right) .
\end{aligned}
$$

Multiplying these equations together by Grassmann's rule, we get $d x^{\prime} d y^{\prime} d z^{\prime}-w_{v}^{\prime} d y^{\prime} d z^{\prime} d t^{\prime}-w_{y}^{\prime} d z^{\prime} d x^{\prime} d t-w_{:}^{\prime} d x^{\prime} d y^{\prime} d t^{\prime}$
$\quad=\left|\begin{array}{lll}\nu_{11} & \nu_{12} & \nu_{13} \\ \nu_{21} & \nu_{22} & \nu_{23} \\ \nu_{31} & \nu_{32} & \nu_{33}\end{array}\right|\left(d x d y d z-w_{x} d y d z d t-w_{1 \prime} d z d x d t-v_{z} d x d y d t\right)$.

This shows that there is an integral invariant of the form

$$
\theta\left(w_{x} d y d z d t+w_{y} d z d x d t+w_{z} d x d y d t-d x d y d z\right) .
$$

This fact has already been used in $\S 3$ and will be required again in $\S 7$. 
We sball now obtain some further properties of a general space time transformation.

When an expression has the same form in the dashed letters as in the original ones it will be convenient to say that it is an invariant.

Let us suppose that the bilinear form

$$
\begin{aligned}
A d x \delta x & +B d y \delta y+C d z \delta z+D d t \delta t+F(d y \delta z+d z \delta y) \\
& +G(d z \delta x+d x \delta z)+H(d x \delta y+\delta x d y)+U(d x \delta t+\delta x d t) \\
& +V(d y \delta t+d t \delta y)+W(d z \delta t+d t \delta z)
\end{aligned}
$$

is an invariant. If 'we multiply it by itself four times according to Grassmann's rule, we obtain the invariant

$$
\left|\begin{array}{llll}
A & H & G & U \\
H & B & F & V \\
G & F & C & W \\
U & V & W & D
\end{array}\right| d x d y d z d t \delta x \delta y \delta z \delta t .
$$

Putting $d x=\delta x, \ldots$, and denoting the determinant by $\Delta$, we bave the invariant

$$
\sqrt{ } \Delta d x d y d z d t
$$

Now, let $\quad u_{x} d y d z d t+u_{y} d z d x d t+u_{z} d x d y d t+u_{t} d x d y d z$

be an integral invariant of the third order.

Multiplying by (1) and rejecting the factor $\sqrt{ } \Delta d x d y d z d t$, we obtain an invariant

$$
v_{x} d x+v_{y} d y+v_{z} d z+v_{t} d t
$$

where

$$
\left.\begin{array}{l}
\sqrt{ } \Delta v_{x}=A u_{x}+H u_{y}+G u_{z}+U u_{t} \\
\sqrt{ } \Delta v_{y}=H u_{x}+B u_{y}+F u_{z}+V u_{t} \\
\sqrt{ } \Delta v_{z}=G u_{x}+F u_{y}+C u_{z}+W u_{t} \\
\sqrt{ } \Delta v_{z}=U u_{x}+V u_{y}+W u_{z}+D u_{t}
\end{array}\right\} .
$$

Multiplying (3) and (4), we obtain the invariant

$$
\begin{aligned}
{\left[A u_{x}^{2}+B u_{y}^{2}+C u_{z}^{2}+D u_{t}^{2}\right.} & +2 F u_{y} u_{z}+2 G u_{z} u_{x}+2 H u_{x} u_{y} \\
& \left.+2 U u_{x} u_{t}+2 V u_{y} u_{t}+2 W u_{z} u_{t}\right] \frac{d x d y d z d t}{\sqrt{ } \Delta} ;
\end{aligned}
$$

hence the quantity in the square brackets divided by $\Delta$ is an invariant.

Conversely, if we are given an integral form of the first order (4), we may obtain a reciprocal integral form of the third order (3), by multiply- 
ing (4) by (1) three times and rejecting the factor $d x d y d z d t \sqrt{ } \Delta$. The relations expressing the quantities $u_{x}$ in terms of the quantities $v_{x}$ are exactly the same as those obtained by solving equations (5).

Next, suppose we are given an integral form of the second order

$$
B_{x} d y d z+B_{y} d z d x+B_{z} d x d y+E_{x} d x d t+E_{y} d y d t+E_{z} d z d t,
$$

which is an invariant. Then multiplying it twice by (1), and rejecting the factor $\sqrt{ } \Delta d x d y d z d t$, we obtain a reciprocal integral invariant

$$
D_{x} d y d z+D_{y} d z d x+D_{z} d x d y-H_{x} d x d t-H_{y} d y d t-H_{z} d z d t
$$

where

$$
\begin{aligned}
& \sqrt{ } \Delta D_{x}=\left(B C-F^{2}\right) E_{r}+(F G-C H) E_{y}+(H F-B G) E_{:} \\
& +(H W-V G) B_{x}+(B W-V F) B_{y}+(F W-C V) B_{z} \\
& \begin{array}{ccccccc}
\cdots & \cdots & \cdots & \cdots & \cdots & \cdots & \cdots \\
-\sqrt{ } \Delta H_{v}= & (H W-V G) E_{x}+(G U-A W) E_{y}+(A V-H U) E_{z}
\end{array} \\
& +\left(A D-U^{2}\right) B_{x}+(H D-U V) B_{y}+(G D-U W) B_{\varepsilon}
\end{aligned}
$$

The relation between these two invariants is evidently a mutual one.

Multiplying them together, we obtain the integral invariant

$$
\left(E_{x} D_{x}+E_{y} D_{y}+E_{z} D_{z}-H_{x} B_{x}-H_{y} B_{y}-H_{z} B_{z}\right) d x d y d z d t
$$

and the absolute invariant

$$
\frac{1}{\Delta}\left[\left(B C-F^{2}\right) E_{x}^{2}+\left(C A-G^{2}\right) E_{y}^{2}+\ldots\right] .
$$

The theory of reciprocal invariants can evidently be extended to the case in which there are $n$ variables, and a bilinear integral form is known to be invariant.

If we suppose that a biquedratic integral form

$$
\begin{aligned}
& \kappa_{11} d y d z \delta y \delta z+\kappa_{22} d z d x \delta z \delta x+\kappa_{33} d x d y \delta x \delta y \\
+ & \kappa_{44} d x d t \delta x \delta t+\kappa_{55} d y d t \delta y \delta t+\kappa_{66} d z d t \delta z \delta t \\
+ & \kappa_{23}(d z d x \delta x \delta y+d x d y \delta z \delta x)+\ldots
\end{aligned}
$$

is invariant for a transformation, we may multiply it by itself and obtain the invariant

$$
\left(\kappa_{11} \kappa_{44}+\ldots+2 \kappa_{28} \kappa_{14}+\ldots\right) d x d y d z d t \delta x \delta y \delta z \delta t .
$$


Putting $d x=\delta x$, and extracting the square root, we obtain an invariant

Now, let

$$
\sqrt{ } \Theta d x d y d z d t \text {. }
$$

$$
B_{x} d y d z+B_{y} d z d x+B_{z} d x d y+E_{x} d x d t+E_{y} d y d t+E_{z} d z d t
$$

be an invariant of the second order. Multiplying it by (12) and rejecting a factor $\sqrt{ } \Theta d x d y d z d t$, we obtain an invariant

$$
D_{x} \delta y \delta z+D_{y} \delta z \delta x+D_{z} \delta x \delta y-H_{x} \delta x \delta t-H_{y} \delta y \delta t-H_{z} \delta z \delta t,
$$

where

$$
\begin{aligned}
\sqrt{ } \Theta D_{x} & =\kappa_{11} E_{x}+\kappa_{12} E_{y}+\kappa_{13} E_{z}+\kappa_{14} B_{x}+\kappa_{15} B_{y}+\kappa_{16} B_{z}, \\
& \ldots \quad \cdots \quad \ldots \quad \cdots \quad \cdots \quad \ldots \quad \cdots \\
-\sqrt{ } \Theta H_{x} & =\kappa_{41} E_{x}+\kappa_{42} E_{y}+\kappa_{43} E_{z}+\kappa_{44} B_{x}+\kappa_{45} B_{y}+\kappa_{46} B_{z} .
\end{aligned}
$$

The relation between the two invariants will be a mutual one if the coefficients $\kappa_{11}, \ldots$ are the elements of an orthogonal matrix.

The Invariants of a Spherical Wave Transformation.

Starting from the fundamental invariants

$$
\begin{gathered}
d \tau^{2}=\lambda^{2}\left[d t^{2}-d x^{2}-d y^{2}-d z^{2}\right], \\
\lambda^{4} d x d y d z d t, \\
A_{x} d x+A_{y} d y+A_{z} d z-\Phi d t, \\
H_{x} d y d z+H_{y} d z d x+H_{z} d x d y+E_{x} d x d t+E_{y} d y d t+E_{z} d z d t, \\
E_{x} d y d z+E_{y} d z d x+E_{z} d x d y-H_{x} d x d t-H_{y} d y d t-H_{z} d z d t, \\
\rho w_{x} d y d z d t+\rho w_{y} d z d x d t+\rho w_{z} d x d y d t-\rho d x d y d z,
\end{gathered}
$$

we may obtain a number of others by the methods of multiplication and reciprocation. It will be sufficient to enumerate these if we mention the equations from which they are derived,

(1 and 6)

$$
\frac{1}{\lambda^{2}}\left[\rho w_{x} d x+\rho w_{y} d y+\rho w_{z} d z-\rho d t\right],
$$

(5 and 4)

$$
\left(E_{x}^{2}+E_{y}^{2}+E_{z}^{2}-H_{x}^{2}-H_{y}^{2}-H_{z}^{2}\right) d x d y d z d t,
$$

(4 and 4)

$$
\left(E_{x} H_{x}+E_{y} H_{y}+E_{z} H_{z}\right) d x d y d z d t,
$$

(3 and 6)

$$
\rho\left[A_{x} w_{s}+A_{y} w_{y}+A_{z} v_{z}-\Phi\right] d x d y d z d t,
$$

(6 and 7)

$$
\frac{\rho^{2}}{\lambda^{2}}\left(1-w^{2}\right) d x d y d z d t
$$


(5 and 7) $\frac{\rho}{\lambda^{2}}\left[\left(E_{x}-w_{z} H_{y}+w_{y} H_{z}\right) d y d z d t+\left(E_{y}-w_{x} H_{z}+w_{z} H_{z}\right) d z d x d t\right.$ $+\left(E_{z}-w_{y} H_{x}+w_{x} H_{y}\right) d x d y d t-\left(w_{x} E_{x}+w_{y} E_{y}+w_{z} E_{z}\right) d x d y d z,(12)$

$$
\begin{aligned}
& \frac{\rho}{\lambda^{4}}\left[\left(E_{x}+w_{y} H_{z}-w_{z} H_{y}\right) \delta x+\left(E_{y}+w_{z} H_{x}-w_{x} H_{z}\right) \delta y\right. \\
& \left.\quad+\left(E_{z}+w_{x} H_{y}-w_{y} H_{x}\right) \delta z-\left(w_{x} E_{x}+w_{y} E_{y}+w_{z} E_{z}\right) \delta t\right]
\end{aligned}
$$

(19 and 2) $\rho d x d y d z d t\left[\left(E_{x}+w_{y} H_{z}-w_{z} H_{y}\right) \delta x+\left(E_{y}+w_{z} H_{x}-w_{x} H_{z}\right) \delta y\right.$

$$
\left.+\left(E_{z}+w_{x} H_{y}-w_{y} H_{x}\right) \delta z-\left(w_{x} E_{x}+w_{y} E_{y}+w_{z} E_{z}\right) \delta t\right] .
$$

These invariants represent quantities which are of considerable importance in the theory of electrons. ${ }^{*}$ Another invariant which is of some importance is obtained in the following way.

Let $\dagger$

$$
\begin{gathered}
w_{1}=\frac{w_{x}}{\sqrt{ }\left(1-w^{2}\right)}, \quad w_{2}=\frac{w_{y}}{\sqrt{ }\left(1-w^{2}\right)}, \quad w_{3}=\frac{w_{z}}{\sqrt{ }\left(1-w^{2}\right)}, \quad w_{4}=\frac{1}{\sqrt{ }\left(1-w^{2}\right)}, \\
d s^{2}=d t^{2}-d x^{2}-d y^{2}-d z^{2},
\end{gathered}
$$

so that $\quad w_{1}=\frac{d x}{d s}, \quad w_{2}=\frac{d y}{d s}, \quad w_{3}=\frac{d z}{d s}, \quad w_{4}=\frac{d t}{d s}$.

Then

$$
\frac{d w_{1}}{d s}=\frac{\dot{w}_{x}}{1-w^{2}}+\frac{w_{x}(w i v)}{\left(1-w^{2}\right)^{2}}
$$

$$
\frac{d^{2} w_{1}}{d s^{2}}=\frac{\ddot{w}_{x}}{\left(1-w^{2}\right)^{\frac{2}{2}}}+\frac{3 \dot{w}_{x}(w \dot{w})}{\left(1-w^{2}\right)^{\frac{3}{3}}}+\frac{w w_{x}}{\left(1-w^{2}\right)^{\frac{3}{3}}}\left\{w \ddot{w}+\frac{3(w \dot{w})^{2}}{1-w^{2}}+\dot{w}^{2}+\frac{(w \dot{w})^{2}}{1-w^{2}}\right\} .
$$

\section{Hence}

$$
\begin{aligned}
\left(\frac{d w_{1}}{d s}\right)^{2}+\left(\frac{d w_{2}}{d s}\right)^{2}+\left(\frac{d w_{3}}{d s}\right)^{2}-\left(\frac{d w_{4}}{d s}\right)^{2} & \\
& =\frac{\dot{w}^{2}}{\left(1-w^{2}\right)^{2}}+\frac{2(w \dot{w})^{2}}{\left(1-w^{2}\right)^{3}}+\frac{w^{2}(w \dot{w})^{2}}{\left(1-w^{2}\right)^{4}}-\frac{(w \dot{w})^{2}}{\left(1-w^{2}\right)^{4}} \\
& =\frac{\dot{w}^{2}}{\left(1-w^{2}\right)^{2}}+\frac{(w \dot{w})^{2}}{\left(1-w^{2}\right)^{3}}
\end{aligned}
$$

* Some of these are already known in the case of Lorentz's transformations. Cf. Planck, Ann. der Physik, Bd. 26, p. 1 (1908). Minkowski, Göttinger Nachrichten (1908), Born. Ann. d. Physik, Bd. 28 (1909).

+ Cf. Minkowski, Gött. Nachr. (1908). 
Thus

$$
\begin{aligned}
\frac{d^{2} w_{1}}{d s^{2}}-w_{1}\left\{\left(\frac{d w_{1}}{d s}\right)^{2}+\right. & \left.\left(\frac{d w_{2}}{d s}\right)^{2}+\left(\frac{d w w_{3}}{d s}\right)^{2}-\left(\frac{d w_{4}}{d s}\right)^{2}\right\} \\
& =\frac{\ddot{w}_{x}}{\left(1-w^{2}\right)^{3}}+\frac{3 \cdot \dot{w}_{x}(w i v)}{\left(1-w^{2}\right)^{2}}+\frac{w_{x}}{\left(1-w^{2}\right)^{\frac{1}{2}}}\left\{(w \ddot{w})+\frac{3\left(w w^{2}\right)^{2}}{1-w^{2}}\right\} .
\end{aligned}
$$

Now Abraham* has given a formula for the reaction of radiation upon a moving electron, the $x$ component of the reaction being

$$
\left.K_{x}=\frac{2 e^{2}}{3\left(1-w^{2}\right)}\left[\ddot{v}_{x}+\dot{w}_{x} \frac{3(w \dot{w})}{1-w^{2}}+\frac{w_{x}}{1-w^{2}} \hat{i}(w \ddot{w})+\frac{3(w \dot{w})^{2}}{1-w^{2}}\right\}\right] ;
$$

accordingly,

$$
\begin{aligned}
K_{x} d t & =\frac{2 e^{2}}{3 c^{2}}\left[\frac{d^{2} w_{1}}{d s^{2}}-w_{1}\left\{\left(\frac{d w_{1}}{d s}\right)^{2}+\left(\frac{d w_{2}}{d s}\right)^{2}+\left(\frac{d w_{8}}{d s}\right)^{2}-\left(\frac{d w_{4}}{d s}\right)^{2}\right\}\right] d s \\
& =K_{1} d s .
\end{aligned}
$$

To complete the symmetry of the result, we define quantities $K_{t}$ and $K_{4}$ by the equations

$$
\begin{aligned}
K_{t} d t & =\frac{2 e^{2}}{3 c^{2}}\left[\frac{d^{2} w_{4}}{d s^{2}}-w_{4}\left\{\left(\frac{d w_{1}}{d s}\right)^{2}+\left(\frac{d w_{2}}{d s}\right)^{2}+\left(\frac{d w_{9}}{d s}\right)^{2}-\left(\frac{d w_{4}}{d s}\right)^{2}\right\}\right] d s \\
& =K_{4} d s
\end{aligned}
$$

we then have

$$
\begin{gathered}
w_{x} K_{x}+w_{y} K_{y}+w_{z} K_{z}=K_{t}, \\
w_{1} K_{1}+w_{2} K_{2}+w_{3} K_{3}+w_{4} K_{4}=0 .
\end{gathered}
$$

Lauet has shown recently that Abraham's formula may be derived by means of the principle of relativity. We shall complete this result by showing that

$$
\left(K_{x} \delta x+K_{y} \delta y+K_{z} \delta z-K \delta t\right) d t
$$

is an integral invariant for the whole group of spherical wave transformations. It will be sufficient to prove this for the case of the transformation

$$
x^{\prime}=\frac{x}{r^{2}-t^{2}}, \quad y^{\prime}=\frac{y}{r^{2}-t^{2}}, \quad z^{\prime}=\frac{z}{r^{2}-t^{2}}, \quad t^{\prime}=\frac{t}{r^{2}-t^{2}} .
$$


1909.] The trangformation of the electrodynamical equations.

Now, if

$$
\begin{gathered}
d s^{\prime}=\frac{d s}{r^{2}}, \quad \rho^{2}=\vartheta^{2}-t^{2}, \\
\frac{d x^{\prime}}{d s^{\prime}}=\left(1-\frac{2 x^{2}}{\rho^{2}}\right) \frac{d x}{d s}-\frac{2 x y}{\rho^{2}} \frac{d y}{d s}-\frac{2 x z}{\rho^{2}} \frac{d z}{d s}+\frac{2 x t}{\rho^{2}} \frac{d t}{d s} \\
\frac{d^{2} x^{\prime}}{d s^{\prime 2}}=\left(\rho^{2}-2 x^{2}\right) \frac{d^{2} x}{d s^{2}}-2 x y \frac{d^{2} y}{d s^{2}}-2 x z \frac{d^{2} z}{d s^{2}}+2 x t \frac{d^{2} t}{d s^{2}} \\
+2 x-2 \frac{d x}{d s} \rho \frac{d \rho}{d s}+4 x\left(\frac{d \rho}{d s}\right)^{2},
\end{gathered}
$$

$$
\begin{aligned}
\frac{d^{3} x^{\prime}}{d s^{\prime}} d s^{\prime}=d s^{\prime}\left[\left(\rho^{2}-2 x^{2}\right) \frac{d^{3} x}{d s^{3}}-2 x y \frac{d^{3} y}{d s^{3}}-2 x z \frac{d^{3} z}{d s^{3}}+2 x t \frac{d^{3} t}{d s^{3}}\right. \\
\left.-4 r \frac{d^{2} r}{d s^{2}} \frac{d x}{d s}+8 x \frac{d r}{d s} \frac{d^{2} r}{d s^{2}}\right] .
\end{aligned}
$$

Hence

$$
\begin{aligned}
& {\left[\frac{d^{3} x^{\prime}}{d s^{\prime 8}}-\frac{d x^{\prime}}{d s^{\prime}}\left\{\left(\frac{d^{2} x^{\prime}}{d s^{\prime 2}}\right)^{2}+\left(\frac{d^{2} y^{\prime}}{d s^{\prime 2}}\right)^{2}+\left(\frac{d^{2} z^{\prime}}{d s^{\prime \prime 2}}\right)^{2}-\left(\frac{d^{2} t^{\prime}}{d s^{\prime 2}}\right)^{2}\right\}\right] d s} \\
& =d s\left[\left(\rho^{2}-2 x^{2}\right)\left\{\frac{d^{3} x}{d s^{3}}-\frac{d x}{d s}\left[\left(\frac{d^{2} x}{d s^{2}}\right)^{2}+\left(\frac{d^{2} y}{d s^{2}}\right)^{2}+\left(\frac{d^{2} z}{d s^{2}}\right)^{2}-\left(\frac{d^{2} t}{d s^{2}}\right)^{2}\right]\right\}\right. \\
& -2 x y\left\{\frac{d^{3} y}{d s^{3}}-\frac{d y}{d s}\left[\left(\frac{d^{2} x}{d s^{2}}\right)^{2}+\left(\frac{d^{2} y}{d s^{2}}\right)^{2}+\left(\frac{d^{2} z}{d s^{2}}\right)^{2}-\left(\frac{d^{2} t}{d s^{2}}\right)^{2}\right]\right\} \\
& -2 x z ! \frac{d^{3} z}{d s^{3}}-\frac{d z}{d s}\left[\left(\frac{d^{2} x}{d s^{2}}\right)^{2}+\left(\frac{d^{2} y}{d s^{2}}\right)^{2}+\left(\frac{d^{2} z}{d s^{2}}\right)^{2}-\left(\frac{d^{2} t}{d s^{2}}\right)^{2}\right] \text {; } \\
& +2 x t\left\{\frac{d^{3} t}{d s^{3}}-\frac{d t}{d s}\left[\left(\frac{d^{2} x}{d s^{2}}\right)^{2}+\left(\frac{d^{2} y}{d s^{2}}\right)^{2}+\left(\frac{d^{2} z}{d s^{2}}\right)^{2}-\left(\frac{d^{2} t}{d s^{2}}\right)^{2}\right] !\right] \text {. } \\
& \text { Now } \quad \frac{\partial x}{\partial x^{\prime}}=\rho^{2}-2 x^{2}, \quad \frac{\partial y}{\partial x^{\prime}}=-2 x y, \quad \ldots \text {; }
\end{aligned}
$$

therefore

$$
\left(K_{x}^{\prime} \delta x^{\prime}+K_{y}^{\prime} \delta y^{\prime}+K_{z}^{\prime} \delta z-K_{t}^{\prime} \delta t^{\prime}\right) d t^{\prime}=\left(K_{x} \delta x+K_{y} \delta y+K_{z} \delta z-K_{t} \delta t\right) d t .
$$

The Electrodynamical Equations for Ponderable Bodies.

We shall consider a system of electrodynamical equations for moving ponderable bodies of the type used by Minkowski, ${ }^{*}$ Einstein and Laub. ${ }^{+}$

* Göttinger Nachrichten (1908).

† Amnalen der Phys., Bd. 26 (1908). 
These equations are of the form

$$
\left.\begin{array}{ll}
\operatorname{curl} H=\frac{\partial D}{\partial t}+s, & \operatorname{div} D=\rho \\
\operatorname{curl} E=-\frac{\partial B}{\partial t}, & \operatorname{div} B=0
\end{array}\right\}
$$

where the vectors $E, H, D, B$ denote the electric force, magnetic force, electric displacement, and magnetic induction respectively, $s$ denotes the current and $\rho$ the volume density of electricity. The equations differ from those used by Lorentz by the fact that the vector $H-[P w]$ occurring in Lorentz's equations is replaced here by the vector $H .^{*}$ It should be remarked that Frank + has obtained Minkowski's equations by a process of averaging in the case of non-magnetic bodies.

We shall suppose that the electric polarisation $P$ and the magnetic polarisation $Q$ are connected with $D, E, B$, and $H$ by the formulæ

$$
P=D-E, \quad Q=B-H .
$$

The electrodynamical equations (I) can be replaced by the two integral equations

$$
\begin{aligned}
& \iint\left(B_{r} d y d z+B_{y} d z d x+B_{z} d x d y+E_{x} d x d t+E_{y} d y d t+E_{z} d z d t\right)=0 \\
& \iint\left(D_{x} d y d z+D_{y} d z d x+D_{z} d x d y-H_{x} d x d t-H_{y} d y d t-H_{z} d z d t\right) \\
& =-\iiint\left(s_{x} d y d z d t+s_{y} d z d x d t+s_{z} d x d y d t-\rho d x d y d z\right) .
\end{aligned}
$$

These equations are unaltered in form by a transformation from $(x, y, z, t)$ to $\left(x^{\prime}, y^{\prime}, z^{\prime}, t^{\prime}\right)$ if

$B_{x} d y d z+B_{y} d z d x+B_{z} d x d y+E_{x} d x d t+E_{y} d y d t+E_{z} d z d t$

$=\theta\left[B_{x}^{\prime} d y^{\prime} d z^{\prime}+B_{y}^{\prime} d z^{\prime} d x^{\prime}+B_{z}^{\prime} d x^{\prime} d y^{\prime}+E_{x}^{\prime} d x^{\prime} d t^{\prime}+E_{y}^{\prime} d y^{\prime} d t^{\prime}+E_{z} d z^{\prime} d t^{\prime}\right]$,

$D_{x} d y d z+D_{y} d z d x+D_{z} d x d y-H_{x} d x d t-H_{y} d y d t-H_{z} d z d t$

$=\phi\left[D_{x}^{\prime} d y^{\prime} d z^{\prime}+D_{y}^{\prime} d z^{\prime} d x^{\prime}+D_{z}^{\prime} d x^{\prime} d y^{\prime}-H_{x}^{\prime} d x^{\prime} d t^{\prime}-H_{y}^{\prime} d y^{\prime} d t^{\prime}-H_{z}^{\prime} d z^{\prime} d t^{\prime}\right]$,

$s_{x} d y d z d t+s_{y} d z d x d t+s_{z} d x d y d t-\rho d x d y d z$

$$
=\phi\left[s_{x}^{\prime} d y^{\prime} d z^{\prime} d t^{\prime}+s_{y}^{\prime} d z^{\prime} d x^{\prime} d t^{\prime}+s_{z}^{\prime} d x^{\prime} d y^{\prime} d t^{\prime}-\rho^{\prime} d x^{\prime} d y^{\prime} d z^{\prime}\right]
$$

where $\theta$ and $\phi$ are constants.

* This simply means that a different definition is adopted for $H$, the object being to retain the symmetry of the equations.

† Ann. d. Phys., Bd. 27, p. 1059 (1908). 
1909.] The trangformation of the electrodynamical equations.

The formulæ of transformation of the type used by Einsten and Laub in the case of Lorentz's transformation are obtained by putting $\theta=\phi=1$. If we use a spherical wave transformation with positive Jacobian, we can deduce the relation

$$
\begin{aligned}
& E_{x} d y d z+E_{y} d z d x+E_{z} d x d y-B_{x} d x d t-B_{y} d y d t-B_{z} d z d t \\
& \quad=E_{x}^{\prime} d y^{\prime} d z^{\prime}+E_{y}^{\prime} d z^{\prime} d x^{\prime}+E_{z}^{\prime} d x^{\prime} d y^{\prime}-B_{x}^{\prime} d x^{\prime} d t^{\prime}-B_{y}^{\prime} d y^{\prime} d t^{\prime}-B_{z}^{\prime} d z^{\prime} d t^{\prime},
\end{aligned}
$$

and this, combined with $(\mathrm{V})$, gives

$$
\begin{aligned}
& P_{x} d y d z+P_{y} d z d x+P_{z} d x d y-Q_{x} d x d t-Q_{y} d y d t-Q_{z} d z d t \\
& =P_{x}^{\prime} d y^{\prime} d z^{\prime}+P_{y}^{\prime} d z^{\prime} d x^{\prime}+P_{z}^{\prime} d x^{\prime} d y^{\prime}-Q_{x}^{\prime} d x^{\prime} d t^{\prime}-Q_{y}^{\prime} d y^{\prime} d t^{\prime}-Q_{z}^{\prime} d z^{\prime} d t^{\prime},
\end{aligned}
$$

which enables us to obtain the formula of transformation of the polarisation vectors.

The constitutive relations are obtained by Einstein and Laub by assuming that all the bodies in the dashed system of coordinates are at rest, and that in this system

$$
D^{\prime}=\epsilon G^{\prime}, \quad B^{\prime}=\mu H^{\prime}, \quad s^{\prime}=\sigma E^{\prime} .
$$

This gives a set of constitutive relations for the case in which a system of bodies are moving with constant velocity $w$.

The constitutive relations that are obtained in this way may be written in the form *

$$
\left.\begin{array}{rl}
D+[w H] & =\epsilon\left\{E+[w B]_{i}^{i}\right. \\
B-[w E] & =\mu\{H-[w D]\} \\
\frac{s_{w}-|w| \rho}{\sqrt{ }\left(1-w^{2}\right)} & =\sigma\left\{E+[w B]_{i w}\right. \\
s_{\bar{w}} & =\frac{\sigma\left\{E+[w B]_{i-w}\right.}{\sqrt{ }\left(1-w^{2}\right)}
\end{array}\right\},
$$

where the suffix $w$ denotes that the component in the direction of $w, \bar{w}$, a component in a direction perpendicular to $w$ is to be taken.

The first two equations are seen to be invariant for the group of spherical wave tranformations when we obtain them in the following way. The expression

$$
\underline{\lambda}\left[\frac{\left.d t-w_{x} d x-w_{y} d y-w_{z} d z\right]}{\sqrt{ }\left(1-w^{2}\right)}\right.
$$

- Minkowski, Göttinger Nachrichten.(1908); Einstein and Laub, Ann. d. Ph!ls. (1908).

SER. 2. VOL. 8. No. 1052 . 
is seen from (1), $\$ 6$, to be an invariant. If we multiply this by the invariants

$$
\begin{aligned}
& B_{x} d y d z+B_{y} d z d x+B_{z} d x d y+E_{x} d x d t+E_{y} d y d t+E_{z} d z d t \\
& D_{x} d y d z+D_{y} d z d x+D_{z} d x d y-H_{x} d x d t-H_{y} d y d t-H_{z} d z d t \\
& H_{x} d y d z+H_{y} d z d x+H_{z} d x d y+D_{x} d x d t+D_{y} d y d t+D_{z} d z d t \\
& E_{x} d y d z+E_{y} d z d x+E_{z} d x d y-B_{x} d x d t-B_{y} d y d t-B_{z} d z d t
\end{aligned}
$$

we obtain the invariants

$$
\begin{aligned}
& \frac{\lambda}{\sqrt{ }\left(1-w^{2}\right)}\left[\left(B_{x}-v_{y} E_{z}+w_{z} E_{y}\right) d y d z d t+\left(B_{y}-w_{z} E_{x}+w_{x} E_{z}\right) d z d x d t\right. \\
& \left.+\left(B_{z}-w_{x} E_{y}+w_{y} E_{x}\right) d x d y d t-\left(w_{x} B_{x}+w_{y} B_{y}+v_{z} B_{z}\right) d x d y d z\right], \\
& \frac{\lambda}{\sqrt{ }\left(1-v^{2}\right)}\left[\left(D_{x}+w_{y} H_{z}-w_{z} H_{y}\right) d y d z d t+\left(D_{y}+w_{z} H_{x}-w_{x} H_{z}\right) d z d x d t\right. \\
& \left.+\left(D_{z}+w_{x} H_{y}-w_{y} H_{x}\right) d x d y d t-\left(w_{x} D_{x}+w_{y} D_{y}+w_{z} D_{z}\right) d x d y d z\right], \\
& \frac{\lambda}{\sqrt{ }\left(1-v^{2}\right)}\left[\left(H_{x}-v_{y} D_{z}+w_{z} D_{y}\right) d y d z d t+\left(H_{y}-w_{z} D_{x}+w_{x} D_{z}\right) d z d x d t\right. \\
& \left.+\left(H_{z}-w_{x} D_{y}+v_{y} D_{x}\right) d x d y d t-\left(v_{x} H_{x}+w_{y} H_{y}+w_{z} H_{z}\right) d x d y d z\right], \\
& \frac{\lambda}{\sqrt{ }\left(1-w^{2}\right)}\left[\left(E_{x}+w_{y} B_{z}-w_{z} B_{y}\right) d y d z d t+\left(E_{y}+w_{z} B_{x}-w_{x} B_{z}\right) d z d x d t\right. \\
& \left.+\left(E_{z}+w_{x} B_{y}-w_{y} B_{x}\right) d x d y d t-\left(w_{. x} E_{x}+w_{y} E_{y}+w_{z} E_{z}\right) d x d y d z\right],
\end{aligned}
$$

and the constitutive relations are obtained by making the first of these equal to $\mu$ times the third, and the second equal to $\epsilon$ times the fourth, where $\epsilon$ and $\mu$ are invariants. These, however, are not the only constitutive relations which remain invariant, ${ }^{*}$ for we may obtain the two integral invariants

$$
\begin{aligned}
& \left(\frac{\partial E_{x}}{\partial t}-\frac{\partial B_{z}}{\partial y}+\frac{\partial B_{y}}{\partial z}\right) d y d z d t+\left(\frac{\partial E_{y}}{\partial t}-\frac{\partial B_{x}}{\partial z}+\frac{\partial B_{z}}{\partial x}\right) d z d x d t \\
& +\left(\frac{\partial E_{z}}{\partial t}-\frac{\partial B_{y}}{\hat{\lambda} x}+\frac{\partial B_{x}}{\partial y}\right) d x d y d t+\left(\frac{\partial E_{x}}{\partial x}+\frac{\partial E_{y}}{\partial y}+\frac{\partial E_{z}}{\hat{\partial} z}\right) d x d y d z ; \\
& \left(\frac{\hat{\imath} H_{1}}{\hat{\imath} t}+\frac{\partial D_{z}}{\partial y}-\frac{\hat{\imath} D_{u}}{\hat{\imath} z}\right) d y d z d t+\left(\frac{\partial H_{y}}{\hat{r} t}+\frac{\partial D_{x}}{\partial z}-\frac{\partial D_{z}}{\partial x}\right) d z d x d t \\
& +\left(\frac{\partial H_{z}}{\partial t}+\frac{\hat{\partial} D_{y}}{\partial x}-\frac{\partial D_{x}}{\partial y}\right) d x d y d t-\left(\frac{\partial H_{x}}{\partial x}+\frac{\partial H_{y}}{\partial y}+\frac{\partial H_{z}}{\partial z}\right) d x d y d z
\end{aligned}
$$

A set of constitutive relations given by two linear relations between 
these six integral forms of the third order is invariant for the group of spherical wave transformations.

To obtain the other constitutive relations, we construct an integral form of the third order reciprocal to $(\mathrm{X})$ and write it in the form

$$
\frac{\rho_{0}}{1-w^{2}}\left[w_{x} d y d z d t+w_{y} d z d x d t+w_{z} d x d y d t-d x d y d z\right],
$$

where $\rho_{0}$ is a quantity which will be determined presently.

Subtracting this from the invariant

$$
s_{x} d y d z d t+s_{y} d z d x d t+s_{z} d x d y d t-\rho d x d y d z,
$$

we may obtain a set of constitutive relations by identifying the resulting invariant with

$$
\begin{aligned}
& \frac{\sigma \lambda}{\sqrt{ }\left(1-w^{2}\right)}\left[\left(E_{x}+w_{y} B_{z}-w_{z} B_{y}\right) d y d z d t+\left(E_{y}+w_{z} B_{y}-w_{x} B_{z}\right) d z d x d t\right. \\
& \left.\quad+\left(E_{z}+w_{x} B_{y}-w_{y} B_{x}\right) d x d y d t-\left(E_{x} w_{x}+E_{y} v_{y}-E_{z} w_{z}\right) d x d y d z\right] .
\end{aligned}
$$

If we multiply the first coefficient in this invariant by $w_{x}$, the second $b y$ $w_{y}$, the third by $w_{z}$, the fourth by 1 , and add, the result is zero. The same must hold in the case of the invariant to which it is equated; therefore

$$
s_{z} w_{x}+s_{y} w_{y}+s_{z} w_{z}-\rho-\frac{\rho_{0}}{1-w^{2}}\left[w_{w}^{2}+w_{y}^{2}+v_{z}^{2}-1\right]=0
$$

or

$$
\rho=\rho_{0}+(s w) \text {. }
$$

Hence we have the constitutive relations

$$
s-\frac{\rho-(s w)}{1-w^{2}} w=\frac{\sigma \lambda}{\sqrt{\left(1-w^{2}\right)}} i E+[w B]_{i}^{\prime},
$$

which agree with those obtained by Minkowski, Einstein and Laub, in the case when $\lambda=1$.

We shall now show that it is possible to construct a set of constitutive relations which are invariant for a much wider class of transformations.

Let the transformation be biuniform within a certain domain of values of $(x, y, z, t)$, and such that the bilinear form

$$
\begin{aligned}
A d x \delta x & +B d y \delta y+C d z \delta z+D d t \delta t+F(d y \delta z+d z \delta y) \\
& +G(d z \delta x+\delta z d x)+H(d x \delta y+d y \delta x)+U(d x \delta t+\delta x d t) \\
& +V(d y \delta t+\delta y d t)+W(d z \delta t+\delta z d t)
\end{aligned}
$$


is an invariant. When we put $d x=\delta x, \ldots$ this implies that a certain quadratic form is an invariant.

Let us suppose that the constitutive relations connecting $B_{x}, B_{y}, B_{z}$, $E_{v}, E_{\eta}, E_{z}$ with $H_{x}, H_{\eta}, H_{z}, D_{v}, D_{\eta}, D_{z}$, are given by the circumstance that the invariant reciprocal to

$$
D_{r} d y d z+D_{\eta} d z d x+D_{:} d x d y-H_{x} d x d t-H_{\eta !} d y d t-H_{z} d z d t
$$

is an invariant multiple of

$$
B_{x} d y d z+B_{y} d z d x+B_{z} d x d y+E_{x} d x d t+E_{y} d y d t+E_{z} d z d t .
$$

This assumption preserves the analogy with the electron equations where the two fundamental integral invariants of the second order are reciprocals with regard to the quadratic form

$$
\lambda^{2}\left[d x^{2}+d y^{2}+d z^{2}-d t^{2}\right] .
$$

In the present case the relations between the two sets of vectors are of the type

$$
\left.\begin{array}{rl}
\kappa \sqrt{ } \Delta B_{x}=- & \left(B C-F^{2}\right) H_{x}-(F G-C H) H_{y}-(H F-B G) H_{z} \\
& +(H W-V G) D_{x}+(B W-V F) D_{y}+(F W-C V) D_{z} \\
\kappa \sqrt{ } \Delta E_{x}= & -(H W-V G) H_{x}-(G U-A W) H_{y}-(A V-H U) H_{z} \\
& +\left(A D-U^{2}\right) D_{x}+(H D-U V) D_{y}+(G D-U W) D_{z} \\
\frac{1}{\kappa} \sqrt{ } \Delta D_{x}= & \left(B C-F^{2}\right) E_{x}+(F G-C H) E_{y}+(H F-B G) E_{z} \\
& +(H W-V G) B_{x}+(B W-V F) B_{y}+(F W-C V) B_{z} \\
-\frac{1}{\kappa} \sqrt{ } \Delta H_{x}= & (H W-V G) E_{x}+(G U-A W) E_{y}+(A V-H U) E_{z} \\
& +\left(A D-U^{2}\right) B_{x}+(H D-U V) B_{y}+(G D-U W) B_{z}
\end{array}\right\},
$$

where $\Delta$ denotes the determinant

$$
\left|\begin{array}{llll}
A & H & G & U \\
H & B & F & V \\
G & F & C & W \\
U & V & W & D
\end{array}\right| .
$$

To obtain the other constitutive relations we start with the assumption that there is an integral invariant of the type*

$\theta\left[w_{x} d y d z d t+w_{y} d z d x d t+w_{z} d x d y d t-d x d y d z\right]$.

* This assumption is justified by the remark made on p. 249 . 
From this we may obtain the reciprocal invariant

where

$$
v_{x} d x+v_{y} d y+v_{z} d z+v_{t} d t
$$

$$
\left.\begin{array}{l}
\sqrt{ } \Delta v_{x}=\theta\left(A w_{x}+H w_{y}+G w_{z}+U\right) \\
\sqrt{ } \Delta v_{y}=\theta\left(H w_{x}+B w_{y}+F w_{z}+V\right) \\
\sqrt{ } \Delta v_{z}=\theta\left(G w_{x}+F w_{y}+C w_{z}+W\right) \\
\sqrt{ } \Delta v_{t}=\theta\left(U w_{x}+V w_{y}+W w_{z}+D\right)
\end{array}\right\} .
$$

Multiplying these and rejecting the invariant factor $\sqrt{ } \Delta d x d y d z d t$, we obtain the invariant

$$
\begin{aligned}
\frac{\theta^{2}}{\Delta}\left[A w_{x}^{2}+B w_{y}^{2}+C w_{z}^{2}+D+2 F w_{y} w_{z}\right. & +2 G w_{z} w_{x}+2 H w_{x} u w_{! \prime} \\
& \left.+2 U w_{x}+2 V w_{y}+2 W w_{z}\right]=\theta .
\end{aligned}
$$

Multiplying

$$
D_{x} d y d z+D_{y} d z d x+D_{z} d x d y+H_{x} d x d t-H_{y} d y d t-H_{z} d z d t
$$

by

$$
v_{x} d x+v_{y} d y+v_{z} d z+v_{t} d t,
$$

we obtain the invariant

$\left(v_{t} D_{x}-v_{y} H_{z}+v_{z} H_{y}\right) d y d z d t+\left(v_{t} D_{y}-v_{z} H_{x}+v_{x} H_{z}\right) d x d y d t$

$$
+\left(v_{t} D_{z}-v_{x} H_{y}+v_{y} H_{x}\right) d x d y d t+\left(v_{x} D_{x}+v_{y} D_{y}+v_{z} D_{z}\right) d x d y d z \text {. (5) }
$$

We now assume that

$s_{x} d y d z d t+s_{y} d z d x d t+s_{z} d x d y d z-\rho d x d y d z$

$$
-\theta\left[w_{x} d y d z d t+w_{y} d z d x d t+w_{z} d x d y d t-d x d y d z\right]
$$

is an invariant multiple of this invariant. This gives the relation

$$
s_{x} v_{x}+s_{y} v_{y}+s_{z} v_{z}+\rho v_{t}-\theta\left[v_{x} w_{x}+v_{y} v_{y}+v_{z} u v_{z}+v_{t}\right]=0,
$$

or

$$
s_{x} v_{x}+s_{y} v_{y}+s_{z} v_{z}+\rho v_{t}=\Theta \sqrt{ } \Delta .
$$

The constitutive relations are of the type

$$
\left.\begin{array}{c}
s_{x}-\theta w_{x}=\sigma\left(v_{t} D_{x}-v_{y} H_{z}+v_{z} H_{y}\right) \\
s_{y}-\theta w_{y}=\sigma\left(v_{t} D_{y}-v_{z} H_{x}+v_{x} H_{z}\right) \\
s_{z}-\theta w_{z}=\sigma\left(v_{t} D_{z}-v_{x} H_{y}+v_{y} H_{x}\right)
\end{array}\right\},
$$

and can be expressed in terms of $E$ and $B$ by means of the relations connecting these quantities with $D$ and $H$. 
With these constitutive relations the equations of electrodynamics are invariant for a very large class of space time transformations. The relations simplify considerably when the quadratic form is of a simple type.

The vanishing of the quadratic form may be supposed to represent the condition that two neighbouring particles are in a position to act on one another.

We shall now verify that in a particular case the constitutive relations obtained in this way agree with the ones obtained by Minkowski, Einstein, and Laub.

Starting with a system at rest, we assume that the invariant quadratic form is

$$
d x^{2}+d ! j^{2}+d z^{2}-\epsilon \mu d t^{2} .
$$

This gives

$$
د=-\epsilon \mu \text {, }
$$

and if we take

$$
\kappa=V^{i}\left(-\frac{\epsilon}{\mu}\right) \text {, }
$$

the relations (2) take the form

$$
B=\mu H, \quad E=\epsilon^{-1} D .
$$

Now make the Lorentzian transformation

$$
x=\frac{x^{\prime}-u t^{\prime}}{\sqrt{ }\left(1-u^{2}\right)}, \quad y=y^{\prime}, \quad z=z^{\prime}, \quad t=\frac{t^{\prime}-u x^{\prime}}{\sqrt{ }\left(1-u^{2}\right)} ;
$$

the quadratic form then becomes

$$
d x^{\prime 2}\left[\frac{1-\epsilon \mu \cdot u^{2}}{1-u^{2}}\right]+d y^{\prime 2}+d z^{\prime 2}+d t^{\prime 2} \frac{u^{2}-\epsilon \mu}{1-u^{2}}-2 u \frac{1-\epsilon \mu}{1-u^{2}} d x^{\prime} d t^{\prime} .
$$

We again have $\Delta=-\epsilon \mu$, and the relations (2) take the form

$$
\begin{aligned}
& B_{y}=\mu H_{x}, \\
& B_{y}=\frac{\epsilon \mu-u^{2}}{\epsilon\left(1-u^{2}\right)} H_{y}+u \frac{\epsilon \mu-1}{\epsilon\left(1-u^{2}\right)} D_{:,}, \\
& B_{z}=\frac{\epsilon \mu-u^{2}}{\epsilon\left(1-u^{2}\right)} H_{z}-u \frac{\epsilon \mu-1}{\epsilon\left(1-u^{2}\right)} D_{y}, \\
& E_{. u}=\frac{1}{\epsilon} D_{x}, \\
& E_{y}=\frac{1-u^{2} \epsilon \mu}{\epsilon\left(1-u^{2}\right)} D_{y}+u \frac{\epsilon \mu-1}{\epsilon\left(1-u^{2}\right)} H_{z}, \\
& E_{z}=\frac{1-u^{2} \epsilon \mu}{\epsilon\left(1-u^{2}\right)} D_{z}-u \frac{\epsilon \mu-1}{\epsilon\left(1-u^{2}\right)} H_{y} .
\end{aligned}
$$


These are equivalent to the relations

$$
\begin{aligned}
B_{x} & =\mu H_{x}, \\
B_{y}+u E_{z} & =\mu\left[H_{y}+u D_{z}\right], \\
B_{z}-u E_{y} & =\mu\left[H_{z}-u D_{y}\right], \\
E_{x} & =\epsilon^{-1} D_{y}, \\
E_{y}-u B_{z} & =\epsilon^{-1}\left[D_{y}-u H_{z}\right], \\
E_{z}+u B_{y} & =\epsilon^{-1}\left[D_{y}+u H_{y}\right],
\end{aligned}
$$

obtained by Minkowski, Einstein, and Laub.

It is also easy to verify that the constitutive relations (7) take the form

$$
\frac{s_{x}-u \rho}{\sqrt{ }\left(1-u^{2}\right)}=\sigma E_{z}, \quad s_{y}=\sigma \frac{E_{y}-u B_{i}}{\sqrt{ }\left(1-u^{2}\right)}, \quad s_{z}=\sigma \frac{E_{:}-u B_{y}}{\sqrt{ }\left(1-u u^{2}\right)},
$$

given by Minkowski, Einstein, and Laub.

We have thus shown that the scheme of constitutive relations indicated by the invariance of a quadratic form agrees with the known scheme of relations in particular cases, and is invariant for any relevant space time transformation which is biuniform in a certain domain.

These relations are not the most general possible, and so the configuration and state of motion under consideration is of a special type, but the relations are sufficiently general for most ordinary purposes.

[Note added October 8th, 1909.]

Spherical wave transformations are not the only ones which can be used to transform problems occurring in the theory of electrons, for there are large classes of transformations which can be applied to particular problems, but cannot be applied to an arbitrary problem. The equations of transformation in this case involve the magnitudes of the electric and magnetic forces occurring in the particular problem.

Let us suppose that the electrodynamical field in a particular problem is of such a nature that the components $\left(E_{x}, E_{y}, E_{z}\right),\left(H_{x}, H_{y}, H_{z}\right)$ of the electric and magnetic forces are connected by the relations

$$
\begin{aligned}
& E_{.,} H_{u}+E_{l !} H_{\eta !}+E_{z} H_{z}=0, \\
& E_{x}^{2}+E_{y}^{2}+E_{z}^{2}=H_{x}^{2}+H_{y}^{2}+H_{z}^{2},
\end{aligned}
$$

and let $\left(s_{x}, s_{y}, s_{z}\right)$ be the components of Poynting's vector. 
264 The trangFormation OF THE ELECTRODYNaMical equations.

Then, if $\alpha_{x}, a_{y}, \alpha_{z}, a, s$ are five functions of $x, y, z, t$ satisfying the relations

$$
\begin{gathered}
u_{x} s_{x}+u_{y} s_{y}+u_{z} s_{z}=\alpha s, \\
s_{x}^{2}+s_{y}^{2}+s_{z}^{2}=s^{2},
\end{gathered}
$$

a transformation of coordinates for which

$$
\begin{aligned}
d x^{2}+d y^{2}+d z^{2}-d t^{2}+\left(a_{x} d x+a_{y} d y+a_{z} d z-a d t\right) & \left(s_{x} d x+s_{y} d y+s_{z} d z-s d t\right) \\
& =\lambda\left(d x^{\prime 2}+d y^{\prime 2}+d z^{\prime 2}-d t^{\prime 2}\right),
\end{aligned}
$$

$\lambda$ being a function of $x^{\prime}, y^{\prime}, z^{\prime}, t^{\prime}$, is in general suitable for the transformation of the problem in question. The above equation implies that, if the velocity of radiation is represented by unity in the first system of co. ordinates, it is also represented by unity in the second system.

[March 15th, 1910.-

The relation $u_{x} s_{x}+u_{y} s_{y}+u_{z} s_{z}=a s$ can be omitted.] 